\title{
Localization of quasiparticles in a disordered vortex
}

\author{
R. Bundschuh*, C. Cassanello, D. Serban ${ }^{\dagger}$, and M.R. Zirnbauer \\ Institut für Theoretische Physik \\ Universität zu Köln, Zülpicher Str. 77, D-50937, Köln, Germany
}

\begin{abstract}
We study the diffusive motion of low-energy normal quasiparticles along the core of a single vortex in a dirty, type-II, s-wave superconductor. The physics of this system is argued to be described by a one-dimensional supersymmetric nonlinear $\sigma$ model, which differs from the $\sigma$ models known for disordered metallic wires. For an isolated vortex and quasiparticle energies less than the Thouless energy $E_{\mathrm{Th}}$, we recover the spectral correlations that are predicted by random matrix theory for the universality class $C$. We then consider the transport problem of transmission of quasiparticles through a vortex connected to particle reservoirs at both ends. The transmittance at zero energy exhibits a weak localization correction reminiscent of quasi-one-dimensional metallic systems with symmetry index $\beta=$ 1. Weak localization disappears with increasing energy over a scale set by $E_{\mathrm{Th}}$. This crossover should be observable in measurements of the longitudinal heat conductivity of an ensemble of vortices under mesoscopic conditions. In the regime of strong localization, the localization length is shown to decrease by a factor of 8 as the quasiparticle energy goes to zero.
\end{abstract}

PACS numbers: 74.40.+k, 74.25.Fy, 72.80.Ng, 73.23.-b

\section{Introduction}

Disordered single-electron systems fall into several universality classes distinguished by their symmetries under time reversal and rotation of the electron spin. In mesoscopic physics three such classes are well known, namely those anticipated by Dyson's 1962 classification [1] of manybody systems with complex interactions. They are called the classes with orthogonal, unitary, or symplectic symmetry. In the ergodic regime, where the relevant time scale is much longer than the time for an electron to diffuse across the system, each universality class is described by a Wigner-Dyson random matrix ensemble with symmetry index $\beta=1,2,4$, respectively.

In the present article we consider a fascinating variant of the theme of disordered electron systems: a single vortex in a disordered type-II conventional s-wave superconductor. A simple picture would model the vortex as a tube-shaped metallic region inside the superconductor. Indeed, a vortex supports quasiparticle excitations, which have normal characteristics and are bound to the vortex core by the pairing gap of the superconductor. It is then natural to ask how the quasiparticles of a disordered vortex fit into the traditional classification scheme.

*Current address: Department of Physics, UCSD, La Jolla, CA 92093-0319, U.S.A.

${ }^{\dagger}$ Current address: Service de Physique Théorique, CE-Saclay, F-91191 Gif-Sur-Yvette, France. 
The first and important message is that the low-energy quasiparticles exceed the traditional scheme and form a separate universality class. This assertion can be motivated as follows. Dyson's classification assumes the absence of symmetries of the particle-hole type. When this assumption is relaxed, seven more universality classes enter. Three of these are realized for a massless Dirac particle moving in a random gauge field [2], and another four have recently been identified [3] in metallic systems in contact with a superconductor. Common to all of these classes is an invariance of the energy (or Dirac) spectrum under inversion $E \mapsto-E$. In the limit $E \rightarrow \infty$, the inversion symmetry becomes irrelevant and conventional Wigner-Dyson statistics is recovered, but for small enough $E$ novel features appear. In particular, the disorder average of the density of states is nonstationary and yet universal.

A vortex is an example of a metallic system with particle-hole symmetry. (Note that this particle-hole symmetry is not a dynamical symmetry but arises as a result of doubling the singleparticle Hilbert space à la Bogoliubov-deGennes, in order to incorporate pairing correlations within the formalism of first quantization.) The magnetic field admitted by the vortex breaks time reversal invariance whereas, if magnetic impurities are absent, the spin of a quasiparticle is conserved. According to the classification scheme of [3], this means that a disordered vortex belongs to symmetry class $C$. Moreover, the supercurrent circulating around a vortex causes the phase shift due to Andreev scattering to vanish on average, so that by the reasoning of [3] the Gaussian random matrix ensemble of type $C$ is expected to be appropriate at low excitation energies.

These predictions have recently been applied to dissipative vortex motion in a quick proposal (for pancake vortices) by Skvortsov and Feigel'man [4]. The basic picture is as follows. Dissipation is due to the transfer of energy from the collective motion to the internal degrees of freedom. In the case of a vortex the relevant internal degrees of freedom are those of the quasiparticles. The rate of energy transfer is largely determined by the statistical properties of the quasiparticle wavefunctions and energies. Therefore, a modification of their statistics is expected to modify the rate of dissipation. Indeed, Skvortsov and Feigel'man argued that the level statistics of type $C$ leads to a dissipative flux-flow conductivity which is weakly anomalous.

Although random matrix theory in its universal form is a powerful and successful concept, it is limited to the ergodic regime of times larger than the diffusion time or, equivalently, energies smaller than the Thouless energy. At short times, or high energies, the diffusive dynamics of a disordered electron system causes deviations from random matrix statistics. This is true for ordinary metals and must equally be true for the quasiparticles of a vortex. Clearly, a complete theory of mesoscopic phenomena needs to encompass both regimes and make the crossover between them theoretically accessible. For the case of ordinary metals, such a theory exists and is in good shape. In its most elegant formulation, it is given by the diffusive nonlinear $\sigma$ model pioneered by Wegner [5] and perfected by Efetov [6]. This is a nonlinear field theory of interacting diffusion modes, which has random matrix theory for its universal low-energy limit.

Is it possible to establish a similar description for the quasiparticles of a disordered vortex? As follows from the above discussion, such a description is of relevance to the microscopic theory of dissipative vortex dynamics. It should also be directly testable by experiment, by measuring the longitudinal heat conductivity to probe the transport properties of the quasiparticles at low energies or temperatures. (Note that electrical conductance measurements are ruled out because the vortex is short cut by the superconducting condensate.)

It is clear that the construction of a field theory for the disordered vortex, encompassing both the diffusive quasiparticle dynamics and its ergodic (or random matrix) long time limit, is a more intricate problem than deriving the nonlinear $\sigma$ model for disordered metals. This is because diffusion in a disordered superconductor is already a nontrivial nonlinear process. The 
relevant kinetic equation was derived by Usadel [7], building on earlier work by Eilenberger [8]. For the special geometry of a single vortex, the solution of the Usadel equation was obtained in Refs. [9], [10] to predict the crude structure of the local density of states of the vortex and its thermodynamic properties.

The Usadel equation is well established as a tool in the research area of disordered superconductors and the proximity effect. However, because of its quasiclassical nature, it leaves out mesoscopic effects that are due to quantum coherence, such as weak localization and mesoscopic fluctuation phenomena. The question then is whether one can improve on the quasiclassical Usadel equation and incorporate the relevant quantum effects which, in the long time limit, give rise to random matrix features. The answer is yes. In an important recent letter, Altland et al. [11 pointed out that the Usadel equation can be interpreted as the saddle point equation for a certain supersymmetric field theory of the type of a nonlinear $\sigma$ model. (The general connection between quasiclassical kinetics and nonlinear $\sigma$ models was first understood by Muzykantskii and Khmel'nitskii [12].) The formalism of Altland et al. also shows how to go further and include quantum interference effects, by computing the fluctuations around the saddle point solution. In the present paper this formalism will be applied to the case of a disordered vortex.

A brief account of the contents of this paper is as follows. Section 2 defines the model and sets up the basic field theoretic formalism, the main step being the introduction of a composite field $Q$ by means of a Hubbard-Stratonovich transformation. In Section 3 , two saddle point approximations are made in sequence. The first of these restricts $Q$ to a nonlinear field space obeying the local constraint $Q^{2}(x)=1$. As usual, this approximation is justified for diffusive systems, where the disorder is at the same time (i) sufficiently strong to cause rapid momentum relaxation (thereby leading to diffusive motion in coordinate space) and (ii) weak enough in order for the system to retain metallic characteristics.

The second saddle point approximation is made in the course of solving for the dynamics of the $Q$ field inside the nonlinear space $Q^{2}=1$. To that end, we expand the full Lagrangian of the theory to obtain the relevant "low-energy" Lagrangian, containing in addition to the usual diffusive (or kinetic) term a linear coupling to the superconducting order parameter, and the minimal coupling to the magnetic field present in the vortex. Such an expansion is valid in the so-called "dirty limit", where the coherence length $\xi_{0}$ (i.e. the length scale of variation of the pairing field) exceeds the elastic mean-free path $\ell$. The equation of motion obtained by varying the resulting action functional turns out to be the Usadel equation. Its solution depends only on the scaling variable $\left(\Delta_{0} / D\right)^{1 / 2} r$, where $r$ is the distance from the axis of the vortex, $\Delta_{0}$ is the magnitude of the pairing gap in the bulk, and $D$ is the diffusion constant of the normal metal. This result follows on dimensional grounds and is in agreement with the well-known fact 13. that the coherence length gets reduced by a factor $\left(\ell / \xi_{0}\right)^{1 / 2}$ in the dirty limit.

Section 1 explores the consequences of our key observation, which is that the solution of the Usadel equation is degenerate. More precisely, the field theory for $Q$ has an exact global symmetry broken only by the energy distance from the Fermi level. (This symmetry is not present in traditional work based on the Usadel equation. We believe that its existence can readily be seen only when the field theoretic formalism is used.) Letting the symmetry group act on the solution of the Usadel equation we get a degenerate manifold of solutions. The "radius" of the manifold is maximal on the axis of the vortex and shrinks to zero with increasing distance, over a scale given by $\left(D / \Delta_{0}\right)^{1 / 2} \sim\left(\xi_{0} \ell\right)^{1 / 2}$. Thus, the symmetry becomes ineffective as we move away from the vortex to the asymptotic region. In this way the symmetry, while being global or constant in space, manages to perform the curious trick of having an effect that is localized in space!

For an isolated vortex of length $L$, the statistics of the quasiparticle levels at energies less 
than the Thouless energy $E_{\mathrm{Th}} \sim D / L^{2}$ is determined by the manifold swept out by the symmetry group when acting on the solution of Usadel's equation. By integrating over it, we recover for the vortex the random matrix prediction for the level statistics of systems in universality class $C$. For higher energies $\left(E>E_{\mathrm{Th}}\right)$, or fixed energy and increasing system size $L$, long wave length fluctuations inside the manifold become important. These are governed by a one-dimensional field theory of the nonlinear $\sigma$ model type. We identify the field space as a Riemannian symmetric superspace of type $D \mathrm{III} \mid C \mathrm{I}$. The field is called $q$.

In Section 5 we turn to the investigation of transport properties of the quasiparticle excitations of the disordered vortex. We imagine the two ends of the vortex (or of an ensemble of vortices, to facilitate experiment) to be connected to infinite particle reservoirs and study the problem of transmission of quasiparticles along the axis of the vortex. We work in a mesoscopic regime, requiring low energy and temperature. This problem is the natural analog of calculating the disorder average of the conductance of a mesoscopic metallic wire. The specific observable we study is a correlation function for the conserved probability current carried by quasiparticles of energy $E$. We call this observable the "transmittance", $\tau(E)$. The coupling to the reservoirs is modeled by open boundary conditions on the field $q$ in the standard way.

We then carry out the usual field theoretic perturbation expansion up to one-loop order. To deal with the symmetry breaking due to finite energy $E$, which causes crossover between universality classes, we introduce a parametrization of the fields akin to that used by Altland, Iida and Efetov [14] in their supersymmetric approach to the crossover problem for small disordered electron systems with orthogonal and unitary symmetry. The result we obtain is

$$
\tau=\frac{\xi_{\text {loc }}}{L}-\operatorname{Re}\left(\frac{\operatorname{coth} \sqrt{i \epsilon}}{\sqrt{i \epsilon}}\right)+\mathcal{O}\left(L / \xi_{\text {loc }}\right),
$$

where $\xi_{\text {loc }}$ has the meaning of a localization length, and $\epsilon$ is the energy measured in units of (roughly speaking) the Thouless energy. For quasiparticles of zero energy, the result simplifies to $\tau=\left(\xi_{\text {loc }} / L\right)-(1 / 3)+\ldots$, which is similar to the conductance of quasi-one-dimensional metallic systems in the Wigner-Dyson universality class of orthogonal symmetry $(\beta=1)$. The second term in the expression for $\tau$ is called the weak localization correction. It disappears in the limit $\epsilon \gg 1$, where our field theory reduces to Efetov's nonlinear $\sigma$ model with symmetry index $\beta=2$.

Finally, in Section 6 we tackle the problem of exponential (or strong) localization, which emerges at lengths $L / \xi_{\text {loc }}>1$. The natural tool for calculating the transmittance in this nonperturbative regime is the "quantum Hamiltonian" of the one-dimensional functional integral. By a standard transfer matrix argument, the exponential decay of the transmittance is determined by the lowest nonzero eigenvalue of the quantum Hamiltonian. Calculating it for an arbitrary energy $\epsilon$ is a problem beyond the scope of this paper. However, in the two limits $\epsilon=0$ and $\epsilon \gg 1$ the quantum Hamiltonian is simply a Laplacian on a symmetric superspace and has a large enough group symmetry to be tractable. By computing the low lying spectrum of the Laplacian we find that the localization length for $\epsilon=0$ is eight times smaller than for $\epsilon \gg 1$.

\section{Model and disorder averaged Green functions}

Our starting point are the Bogoliubov-deGennes (BdG) equations for the stationary quasiparticle excitations [13] of a superconductor in (BCS) mean field approximation:

$$
\begin{aligned}
H_{0} u+\Delta v & =E u, \\
\Delta^{*} u-H_{0}^{T} v & =E v .
\end{aligned}
$$


The single-particle Hamiltonian $H_{0}$ is expressed by

$$
H_{0}=(-i \nabla-e A)^{2} / 2 m+V(x)-\mu,
$$

where $\mu$ is the chemical potential (Fermi level) and $A$ is the magnetic vector potential. The functions $u(x)$ and $v(x)$ describe the particle and hole components of a quasiparticle, and are coupled by the pairing potential $\Delta(x)$. The potential energy $V(x)$ is taken to be a Gaussian white noise random potential with zero mean and variance

$$
\langle V(x) V(y)\rangle=v^{2} \delta(x-y) \equiv \frac{1}{2 \pi \nu \tau} \delta(x-y),
$$

where $\nu$ is the density of states and $\tau$ is the elastic mean free time. It is convenient to assemble $H_{0}$ and $\Delta$ into a single operator $H$, here called the BdG "Hamiltonian" for short:

$$
H=\left(\begin{array}{cc}
H_{0} & \Delta \\
\Delta^{*} & -H_{0}^{T}
\end{array}\right),
$$

which acts on the tensor product of $\mathbb{C}^{2}$ with the Hilbert space of square-integrable functions on the coordinate space, $S$, of the superconductor. The factor $\mathbb{C}^{2}$ will be referred to as the particle-hole $(p h)$ space.

The BdG Hamiltonian has the following symmetry property:

$$
H=-\mathcal{C} H^{T} \mathcal{C}^{-1} \quad \text { with } \quad \mathcal{C}=\left(\begin{array}{cc}
0 & 1 \\
-1 & 0
\end{array}\right)
$$

where $T$ means transposition in both particle-hole and coordinate space. As a result of this symmetry, the eigenvalues of $H$ occur in pairs with opposite sign, and we have

$$
\begin{aligned}
G(z) & :=(H-z)^{-1}=\left(-\mathcal{C} H^{T} \mathcal{C}^{-1}-z\right)^{-1} \\
& =-\mathcal{C}\left((H+z)^{-1}\right)^{T} \mathcal{C}^{-1}=-\mathcal{C} G^{T}(-z) \mathcal{C}^{-1}
\end{aligned}
$$

so that the advanced and retarded Green functions are related by

$$
G_{\alpha \alpha^{\prime}}^{-}\left(x, x^{\prime} ; E\right)=-\sum_{\beta \beta^{\prime}} \mathcal{C}_{\alpha \beta} \mathcal{C}_{\alpha^{\prime} \beta^{\prime}} G_{\beta^{\prime} \beta}^{+}\left(x^{\prime}, x ;-E\right) .
$$

To compute disorder averaged Green functions, we will use Efetov's supersymmetry method [6], in its recent adaptation by Altland et al. [11] to include the pairing correlations of a superconductor. In this method, disorder averages of Green functions are obtained from a generating functional

$$
\mathcal{Z}[j]=\left\langle\int \mathcal{D} \bar{\psi} \mathcal{D} \psi \exp \int_{S} d^{3} x(i \bar{\psi}(H-z) \psi+\bar{\psi} j+\bar{j} \psi)\right\rangle .
$$

The functional integral is over supervectors $\psi$ and $\bar{\psi}$, whose components are commuting and anticommuting (or Grassmann) fields [6]. Thus, in addition to the (physical) particle-hole space, an auxiliary "boson-fermion" ( $b f$ ) space is introduced. To average a product of $n$ Green functions, we further enlarge the field space by forming the tensor product with $\mathbb{C}^{n}$ (i.e. we take $n$ copies of the field space). We do not distinguish between "advanced" and "retarded" fields, as we shall make use of the aforementioned symmetry relating $G^{-}$with $G^{+}$. 
To take advantage of the particle-hole symmetry of the BdG Hamiltonian, we find it convenient to tensor the field space with yet another factor $\mathbb{C}^{2}$. This extra "spin" degree of freedom, referred to by the name of "charge conjugation" ( $c c)$ space, is introduced by rearranging the quadratic form of the generating functional as follows:

$$
\begin{aligned}
2 \bar{\psi}(H-z) \psi & =\bar{\psi}(H-z) \psi+\psi^{T}\left(H^{T}-z\right) \bar{\psi}^{T} \\
& =\bar{\psi}(H-z) \psi+\psi^{T}\left(-\mathcal{C}^{-1} H \mathcal{C}-z\right) \bar{\psi}^{T} \\
& =\left(\begin{array}{ll}
\bar{\psi} & -\psi^{T} \mathcal{C}^{-1}
\end{array}\right)\left(\begin{array}{cc}
H-z & 0 \\
0 & H+z
\end{array}\right)\left(\begin{array}{c}
\psi \\
\mathcal{C} \bar{\psi}^{T}
\end{array}\right) .
\end{aligned}
$$

The superscript $T$ here denotes the supertransposition operation. If we define

$$
\Psi=\frac{1}{\sqrt{2}}\left(\begin{array}{c}
\psi \\
\mathcal{C} \bar{\psi}^{T}
\end{array}\right), \quad \bar{\Psi}=\frac{1}{\sqrt{2}}\left(\begin{array}{ll}
\bar{\psi} & -\psi^{T} \mathcal{C}^{-1}
\end{array}\right),
$$

we have

$$
\bar{\psi}(H-z) \psi=\bar{\Psi}(H-\omega) \Psi,
$$

where $\omega=\Sigma_{3} \otimes z$. We use the notation $\Sigma_{i}$ for the Pauli matrices acting in the charge conjugation space and $\sigma_{i}$ for the ones acting in the particle-hole space. As is easily checked, the two supervectors $\bar{\Psi}, \Psi$ obey the symmetry relations

$$
\Psi=\mathcal{C} \gamma \bar{\Psi}^{T}, \quad \bar{\Psi}=-\Psi^{T} \mathcal{C}^{-1} \gamma^{-1},
$$

where $\gamma$ is defined by

$$
\gamma=1_{p h} \otimes\left(E_{B B} \otimes \Sigma_{1}-E_{F F} \otimes i \Sigma_{2}\right) \otimes 1_{n}
$$

with $E_{B B}\left(E_{F F}\right)$ being the projector on the bosonic (fermionic) space. Note that $\gamma$ and $\mathcal{C}$ commute. The trick of doubling $\psi$ to $\Psi$ will help to make the symmetries of the nonlinear $\sigma$ model manifest at all stages.

To summarize, the generating functional for averages of products of Green functions can be written as

$$
\mathcal{Z}[J]=\left\langle\int \mathcal{D} \bar{\Psi} \mathcal{D} \Psi \exp \int_{S} d^{3} x(i \bar{\Psi}(H-\omega) \Psi+\bar{\Psi} J+\bar{J} \Psi)\right\rangle
$$

with sources $J, \bar{J}$ that obey the same symmetry relations as $\Psi, \bar{\Psi}$. The structure of the operators in this expression is the following:

$$
\begin{aligned}
H & =H_{p h} \otimes 1_{b f} \otimes 1_{c c} \otimes 1_{n}, \\
\omega & =1_{p h} \otimes 1_{b f} \otimes \Sigma_{3} \otimes \operatorname{diag}\left(z_{1}, \ldots, z_{n}\right)
\end{aligned}
$$

where $z_{j}=E_{j}-i \eta_{j}$ are complex numbers with negative imaginary parts $\left(-\eta_{j}<0\right)$. In using this tensor product notation, we will usually omit trivial factors of unity.

After this digression about notation, we go about deriving the effective field theory for the disordered superconductor. First of all, decomposing $H$ into regular and stochastic parts as $H=\mathcal{H}_{0}+V(x) \sigma_{3}$, we perform the average over disorder in the generating functional $\mathcal{Z}$ :

$$
\begin{aligned}
\mathcal{Z}[0] & =\int \mathcal{D} \bar{\Psi} \mathcal{D} \Psi \int \mathcal{D} V \exp \int d^{3} x\left(i \bar{\Psi}\left(\mathcal{H}_{0}-\omega\right) \Psi+i \bar{\Psi} \sigma_{3} \Psi V(x)-\frac{V^{2}(x)}{2 v^{2}}\right) \\
& =\int \mathcal{D} \bar{\Psi} \mathcal{D} \Psi \exp \int d^{3} x\left(i \bar{\Psi}\left(\mathcal{H}_{0}-\omega\right) \Psi-\frac{v^{2}}{2}\left(\bar{\Psi} \sigma_{3} \Psi\right)^{2}\right) .
\end{aligned}
$$


The next step is to make a Hubbard-Stratonovich transformation, which allows to extract the relevant "slow modes" of the problem. We are interested in keeping the diffusive modes, i.e. those modes that arise from two-particle channels undergoing multiple scattering with the exchange of momenta smaller than the inverse of the elastic mean-free path, $\ell=v_{F} \tau$. Isolating these modes is a standard procedure [6] which is conveniently performed in Fourier space and has been included in Appendix A for completeness. It is shown there that the contribution from the slow modes to $-v^{2}\left(\bar{\Psi} \sigma_{3} \Psi\right)^{2} / 2$ can be written as

$$
-v^{2} \sum_{|q|<q_{0}} \operatorname{STr} \zeta(-q) \zeta(q)
$$

where $q_{0}=1 / \ell$, and

$$
\zeta(q)=\sum_{k} \Psi(k) \bar{\Psi}(-k+q) \sigma_{3} .
$$

One might think that the magnetic term in the Hamiltonian $\mathcal{H}_{0}$ suppresses the slow modes that couple to the vector potential $A$. It will turn out, however, that the magnetic field of a vortex is not strong enough to cause complete suppression of these modes. Therefore, we will keep all the slow modes appearing in the expression for $\zeta(q)$.

To enable the treatment of the quartic term, one now introduces a Hubbard-Stratonovich like decoupling. This procedure reduces the quartic terms to bilinears, at the expense of introducing additional fields $Q$ :

$$
\begin{aligned}
& \exp \left(-v^{2} \sum_{q} \mathrm{~S} \operatorname{Tr} \zeta(q) \zeta(-q)\right)= \\
& \int \mathcal{D} Q \exp \sum_{q}\left(\frac{\pi \nu}{8 \tau} \mathrm{S} \operatorname{Tr} Q(q) Q(-q)-\frac{1}{2 \tau} \mathrm{S} \operatorname{Tr} Q(q) \zeta(-q)\right) .
\end{aligned}
$$

The symmetry relation

$$
\begin{aligned}
\mathrm{S} \operatorname{Tr} Q \Psi \bar{\Psi} \sigma_{3} & =\mathrm{S} \operatorname{Tr} \sigma_{3} \bar{\Psi}^{T} \Psi^{T} Q^{T}=\operatorname{STr} \sigma_{3}\left(\gamma^{-1} \mathcal{C}^{-1} \Psi\right)(-\bar{\Psi} \gamma \mathcal{C}) Q^{T} \\
& =-\mathrm{S} \operatorname{Sr} \mathcal{C} \gamma Q^{T} \gamma^{-1} \sigma_{3} \mathcal{C}^{-1} \Psi \bar{\Psi}=\operatorname{STr} \sigma_{1} \gamma Q^{T} \gamma^{-1} \sigma_{1} \Psi \bar{\Psi} \sigma_{3}
\end{aligned}
$$

is accounted for by subjecting the supermatrix $Q$ to the linear condition

$$
Q=\sigma_{1} \gamma Q^{T} \gamma^{-1} \sigma_{1} .
$$

After integrating out the quasiparticle fields $\Psi, \bar{\Psi}$ and switching back to the coordinate representation, we obtain

$$
\begin{aligned}
\mathcal{Z}[0] & =\int \mathcal{D} Q \exp -\mathcal{S}[Q], \\
\mathcal{S}[Q] & =-\int d^{3} x\left(\frac{\pi \nu}{8 \tau} \mathrm{S} \operatorname{Tr} Q^{2}-\frac{1}{2} \mathrm{~S} \operatorname{Tr} \ln \left(\mathcal{H}_{0}-\omega+i \sigma_{3} Q / 2 \tau\right)\right) .
\end{aligned}
$$

The integration domain for $\Psi$ and $Q$ has to be chosen with care, in order to ensure the convergence of all integrals. A detailed discussion how to choose integration manifolds correctly can be found in [15].

In summary, the problem of computing disorder averaged Green functions has been reduced to considering the Euclidean field theory with effective action $\mathcal{S}[Q]$. The sign difference compared to Efetov's expression for the effective action comes from the different definition of the supertrace. 


\section{Saddle point approximations}

The next step in deriving the nonlinear $\sigma$ model is to find the saddle points of the effective action (3), and to incorporate the fluctuations around these. Unfortunately, because the gap function $\Delta$ varies in space, the full saddle point equation is too complicated to solve directly. However, in the dirty limit $\tau \Delta \ll 1$, the minimum of the action functional can be found by a two-step procedure 11. In that case the scales set by the disorder and by the superconducting order parameter are well separated, so that one can perform two minimizations in sequence.

To outline this section, the strategy adopted from [11] is as follows. At first, one neglects the gap function $\Delta$ and the deviation $\omega$ of the energy from the Fermi level. One varies the resulting effective action and finds the corresponding saddle point manifold. Then, fluctuations inside this manifold are considered; they couple to the gap function and to the energies $\omega$. The resulting low-energy effective action is varied once again inside the first (high-energy) saddle point manifold. The partial differential equation obeyed by the optimal configuration turns out to coincide with the equation derived by Usadel [7] for the Gorkov Green function of a superconductor in the dirty limit.

\subsection{First saddle point}

Let us write the BdG Hamiltonian for a clean field-free superconductor as

$$
\left.\mathcal{H}_{0}\right|_{A=0}=h_{0} \sigma_{3}+\tilde{\Delta}, \quad \tilde{\Delta}=(\operatorname{Re} \Delta) \sigma_{1}-(\operatorname{Im} \Delta) \sigma_{2},
$$

where $h_{0}=-\nabla^{2} / 2 m-\mu$. In the absence of the gap and at the Fermi energy, variation of the action functional $\mathcal{S}[Q]$ gives the following saddle point equation:

$$
Q(x)=\frac{i}{\pi \nu}\left\langle x\left|\left(h_{0}+i \eta \sigma_{3} \Sigma_{3}+i Q / 2 \tau\right)^{-1}\right| x\right\rangle,
$$

where we kept the positive infinitesimal $\eta$, which is crucial for distinguishing between the physical and unphysical solutions. We assume $\mu \gg 1 / \tau$. Then, since $\left\langle x\left|\left(h_{0}+i \eta\right)^{-1}\right| x\right\rangle$ is understood as $-i \pi \nu$, equation (㺼) is formally solved by a diagonal matrix $Q=\operatorname{diag}\left(q_{1}, q_{2}, \ldots\right)$, with $q_{i}= \pm 1$. To choose the signs correctly, we note that the expression on the right-hand side of the saddle point equation relates to the Green function for the disordered system in the self-consistent Born approximation. The disorder preserves the causal (i.e. retarded versus advanced) character of the Green function, and therefore the sign of $q_{i}$ must coincide with the sign of the imaginary part of the energy. This singles out the solution

$$
Q_{0}=\sigma_{3} \Sigma_{3}
$$

\subsection{Fluctuations around the first saddle point}

In the limit $\tilde{\Delta} \rightarrow 0, A \rightarrow 0$ and $\omega \rightarrow 0$, the action (3) is invariant under the transformation $Q(x) \mapsto T Q(x) T^{-1}$, with $T$ any supermatrix that is constant in space and consistent with the symmetry condition (2) for $Q$. Obviously, the saddle point equation must have the same invariance, and its solution will therefore be degenerate. Indeed, the saddle points lie on a manifold that can be parametrized by $Q=T Q_{0} T^{-1}$, satisfying $Q^{2}=1$. For $\mu \tau \gg 1$ the lowenergy excitations are given by slow transverse fluctuations preserving the constraint $Q^{2}(x)=$ $Q_{0}^{2}=1$. To incorporate these, we put $Q(x):=T(x) Q_{0} T^{-1}(x)$ where $T(x)$ is determined only up 
to "gauge" transformations $T(x) \mapsto T(x) h(x)$ that leave $Q_{0}$ fixed: $h(x) Q_{0} h^{-1}(x)=Q_{0}$. When taken modulo such gauge transformations, the matrix $T(x)$ varies slowly with $x$. Fluctuations that are not of this form are massive and will hence be neglected.

We now insert $Q(x)=T(x) Q_{0} T^{-1}(x)$ into the expression (3) for $\mathcal{S}[Q]$ and expand with respect to $\tilde{\Delta}$ and $\omega$, and up to second order in gradients of $Q(x)$, neglecting higher-order derivatives. In this way the following low-energy effective action governing the transverse fluctuations of $Q$ is obtained:

$$
S[Q]=-\frac{\pi \nu}{8} \int d^{3} x \operatorname{STr}\left(D\left(\nabla_{A} Q\right)^{2}-4 i(\tilde{\Delta}+\omega) \sigma_{3} Q\right) .
$$

Here, $D=v_{F}^{2} \tau / 3$ is the diffusion constant of the normal metal and $\nabla_{A}=\nabla-i e A\left[\sigma_{3}, \cdot\right]$. The details of this calculation can be found in Appendix B.

It will turn out to be of importance that, for $\omega=0$ (and any $A$ and $\tilde{\Delta}$ ), the action functional $S[Q]$ is invariant under transformations

$$
Q(x) \mapsto T Q(x) T^{-1}, \quad \text { if } \quad T=1_{p h} \otimes t
$$

and $t$ is constant in space and obeys $t=\gamma\left(t^{-1}\right)^{T} \gamma^{-1}$. The latter condition means that $t$ runs through an orthosymplectic Lie supergroup $\operatorname{OSp}(2 n \mid 2 n)$.

\subsection{Second saddle point: Usadel equation}

We now want the optimal configuration of the superfield $Q$ for a nonvanishing order parameter $\tilde{\Delta}$ and magnetic vector potential $A$. To find it, we require $S[Q]$ to be stationary with respect to variations of $Q$ that preserve the constraint $Q^{2}=1$. Such variations can be parametrized by

$$
\delta Q(x)=\epsilon[X(x), Q(x)],
$$

where $X$ satisfies $X=-\sigma_{1} \gamma X^{T} \gamma^{-1} \sigma_{1}$ so as to preserve the symmetry (2). The stationarity condition $\delta S=0$ then yields

$$
D \nabla_{A}\left(Q \nabla_{A} Q\right)+i\left[Q,(\tilde{\Delta}+\omega) \sigma_{3}\right]=0 .
$$

With proper interpretation given to $Q$, this coincides with Usadel's equation [7] for the Gorkov Green function - a fact first understood in [11]. By a slight abuse of terminology we shall refer to (7) also as the Usadel equation.

Our plan now is as follows. In the remainder of the current subsection, we are going to solve the Usadel equation for a vortex with cylindrical symmetry (at $\omega=0$ ). To do that, we will introduce cylindrical coordinates $(r, \varphi, z)$ and look for a $z$-independent solution $Q_{0}(r, \varphi)$. Because of the invariance (6), this solution is degenerate and, once again, we are dealing with a manifold of saddle points $T Q_{0}(r, \varphi) T^{-1}$, generated by the action of $T \in \operatorname{OSp}(2 n \mid 2 n)$. In the next section, we will argue that the low-energy excitations of that manifold are given by $Q(x)=$ $T(z) Q_{0}(r, \varphi) T^{-1}(z)$, with $T(z)=1_{p h} \otimes t(z)$. The fluctuations in the $r$ and $\varphi$ directions are strongly suppressed by the coupling to the pairing gap of the superconductor and by transverse quantization. By inserting the last form of $Q(x)$ into the effective action (5) we will obtain yet another effective action, this time for the matrix $T(z)$.

The first step is to solve the Usadel equation at $\omega=0$. This was done before in [9], and we redo it here in a way tailored to our purposes. Recall, first of all, the invariance of the Usadel equation (at $\omega=0$ ) under orthosymplectic transformations $Q(x) \mapsto T Q(x) T^{-1}$, with 
$T=1_{p h} \otimes t$ and $t \in \operatorname{OSp}(2 n \mid 2 n)$. Now, far from the vortex, any solution with finite action $S[Q]$ must become covariantly constant $\left(\nabla_{A} Q \rightarrow 0\right)$ and adjust to the pairing gap $\tilde{\Delta}=|\Delta| \sigma_{1} \mathrm{e}^{-i \varphi \sigma_{3}}$ of the superconductor in the bulk $\left(\left[Q, \tilde{\Delta} \sigma_{3}\right] \rightarrow 0\right)$. These conditions in conjunction with $Q^{2}=1$ determine $Q$ at infinity to be $Q_{\infty}=\sigma_{2} \mathrm{e}^{-i \varphi \sigma_{3}}$, up to multiplication by a sign. Note that $Q_{\infty}$ is stable under symmetry transformations: $Q_{\infty}=\left(1_{p h} \otimes t\right) Q_{\infty}\left(1_{p h} \otimes t\right)^{-1}$. On the other hand, on approaching the vortex core, the unique value of $Q_{\infty}$ is expected to expand into a manifold of solutions of the Usadel equation, as a result of the orthosymplectic invariance. To remove the resulting degeneracy, we hold the solution on the axis of the vortex $(r=0)$ fixed at the metallic saddle point $Q_{0}=\sigma_{3} \otimes \Sigma_{3}$. Given the two limiting forms at $r=0$ and $r=\infty$, it is natural to look for a solution of the form

$$
Q(r, \varphi)=g(r) \sigma_{3} \otimes \Sigma_{3}+f(r) \sigma_{2} \mathrm{e}^{-i \varphi \sigma_{3}} \otimes 1_{c c},
$$

which interpolates between $Q_{0}$ and $Q_{\infty}$. Note that this ansatz is compatible with the symmetry condition (2). To fulfill the constraint $Q^{2}=1$, the functions $g(r)$ and $f(r)$ are required to satisfy

$$
f^{2}(r)+g^{2}(r)=1 .
$$

Note also that $f(r)$ must vanish linearly at $r=0$ in order for $Q(r, \varphi)$ to be nonsingular there.

Next, we insert our ansatz into the Usadel equation, thereby obtaining a differential equation for $g(r)$ and $f(r)$. As is shown in Appendix Q, this equation reads

$$
\frac{1}{r} \partial_{r}\left(r f \partial_{r} g-r g \partial_{r} f\right)+\left(\frac{1}{r}-2 e A_{\varphi}\right)^{2} f g=\frac{2}{D}|\Delta| g .
$$

To complete the formulation of the problem, we need to specify the gap function $|\Delta(r)|$ and the $\varphi$-component of the magnetic vector potential, $A_{\varphi}(r)$. In principle these quantities are to be calculated self-consistently [10] from the function $f(r)$. It is common knowledge, however, that their essential features are as follows. (i) The gap function vanishes on the symmetry axis of the vortex, increases linearly with $r$ for small $r$, and saturates at the bulk value of the superconductor over a scale given by the coherence length $\xi$. (ii) The magnetic field carried by the vortex integrates to one fluxon $h / 2 e$ and is localized in a region of linear size equal to the penetration depth $\lambda$.

For simplicity, we do not insist on an exact self-consistent solution but adopt the following closed-form approximate expressions [16] (for an exact treatment, see Ref. [10]):

$$
2 e A_{\varphi}(r)=\frac{1}{r}-\frac{K_{1}(r / \lambda)}{\lambda}, \quad|\Delta(r)|=\Delta_{0} \frac{r / \xi}{\sqrt{(r / \xi)^{2}+a^{2}}},
$$

which capture the features listed above. The parameter $a$ is a numerical constant whose value will be specified later on. Notice that the vector potential $A$ is everywhere nonsingular, as the Bessel function $K_{1}(r / \lambda)$ behaves as $\lambda / r$ near $r=0$ to cancel the pole $1 / r$. Note also that, given the value of the gap $\Delta_{0}$ far from the vortex, the coherence length $\xi$ no longer is a parameter at our disposal. Rather, $\xi$ has to be determined self-consistently: because $\Delta$ is given by $f$ through the BCS gap equation, the length scale $\xi$ fed into the differential equation (9) must agree with the characteristic scale of the solution emerging from that equation.

To proceed, we solve the constraint $f^{2}+g^{2}=1$ by setting $f=\sin \theta$ and $g=\cos \theta$. If we also introduce the dimensionless variable $u=r / \xi$, the equation for $\theta(u)$ takes the form

$$
-\frac{1}{u} \frac{d}{d u}\left(u \frac{d \theta}{d u}\right)+\left(\frac{K_{1}(u / \kappa)}{\kappa}\right)^{2} \frac{\sin 2 \theta}{2}=\frac{2 \xi^{2} \Delta_{0}}{D} \times \frac{u \cos \theta}{\sqrt{u^{2}+a^{2}}}
$$


with $\kappa=\lambda / \xi$. Let us now specialize to the case of an extreme type-II superconductor, where $\kappa \gg 1$ and the Bessel function can be approximated by $K_{1}(u / \kappa) / \kappa \approx 1 / u$. In this limit, all explicit length scales are removed from the equation - and the self-consistency requirement of equal input and output length scales is satisfied - by setting $2 \xi^{2} \Delta_{0} / D=$ const. Without loss of generality we take the numerical constant to be unity, so that

$$
\xi=\sqrt{D / 2 \Delta_{0}} .
$$

(Note that a different choice of numerical constant would only amount to a redefinition of the parameter a.) This result says that disorder reduces the "clean" value of the coherence length $\xi_{0}=v_{F} / \pi \Delta_{0}$ to a "dirty" value $\xi \sim\left(\ell \xi_{0}\right)^{1 / 2}$. The Usadel equation then simplifies to

$$
-\theta^{\prime \prime}-\frac{\theta^{\prime}}{u}+\frac{\sin 2 \theta}{2 u^{2}}=\frac{u \cos \theta}{\sqrt{u^{2}+a^{2}}} .
$$

As was stated before, the boundary conditions are $\cos \theta(\infty)=g(\infty)=0$ and $\sin \theta(0)=f(0)=0$. Without loss of generality we put $\theta(0)=0$.

For large values of $u$, we can get an approximate analytical solution as follows. The boundary condition at infinity admits the possible values $\theta(\infty)=(2 m+1) \pi / 2$ with $m$ an integer. Writing $\theta(u)=(2 m+1) \pi / 2+h(u)$ and approximating $\sin h$ by $h$ in the asymptotic regime, we get

$$
h^{\prime \prime}+h^{\prime} / u=(-1)^{m} h .
$$

The solutions of this second-order differential equation are combinations of Bessel functions. For even $m$, the solution that goes to zero at infinity behaves asymptotically as

$$
h(u) \sim u^{-1 / 2} \mathrm{e}^{-u},
$$

while for odd $m$, the solutions have the asymptotic form

$$
h(u) \sim u^{-1 / 2} \cos \left(u+u_{0}\right) .
$$

When inserted into the functional (5), the latter solutions can be shown to produce a radially integrated Lagrangian density

$$
\int_{0}^{\infty} g^{2}(r) r d r d \varphi \sim \int d r
$$

which diverges linearly. On the other hand, the solutions for even $m$, which decay exponentially, have a finite radial action. Hence the "good" boundary values at infinity are $\theta(\infty)=\pi / 2+2 \pi n$ with integer $n$. This conclusion is confirmed by a second argument: in Appendix D we show that only the solutions with $\tan \frac{1}{2} \theta(\infty)>0$ can be analytically continued to the normal metal solution, which has to emerge at energies large compared to the gap $\Delta_{0}$.

Since these solutions with $n \in \mathbb{Z}$ are local extrema (and, in fact, minima) of the action, one might naively think that all of them have to be taken into account, according to the principles of saddle point evaluation of integrals. However, the solutions with $n \neq 0$ do not appear anywhere in the large body of literature on the Usadel equation and its applications. What's the reason? The answer is that in the conventional kinetic theory the Usadel equation is based on, one argues that the solutions with $n \neq 0$ are in conflict with causality. Indeed, $g(r)$ has an interpretation [17] as the local density of states (LDoS) at radius $r$, normalized to the density of states of the normal metal. By the properties of the cosine, the solution for $n=0$ is expected to yield a function $g=\cos \theta$ that is everywhere positive, but for $n \neq 0$ regions of negative value inevitably 
appear. In other words, the $n \neq 0$ solutions fail to meet the physical requirement of positivity of the density of states, or causality of the Green function.

If this argument does not seem totally convincing - on the grounds that only the total (not the partial) LDoS is required to be positive - there exists also a functional integral reason why the $n \neq 0$ solutions must be discarded. A careful look reveals that for $\theta(\infty)=\pi / 2+2 \pi n$ the field $Q$ winds $n$ times around the singularity of the logarithm in equation (3). Consequently, the solutions with $n \neq 0$ are not smoothly connected to any choice of integration manifold [15] for $Q$ (before saddle point approximation) that is permitted by the required convergence of all integrals. Put differently, the solutions with $n \neq 0$ are not accessible by deformation of the integration manifold without moving across singularities of the integrand. This uniquely singles out the solution with $n=0$. In summary, we adopt the two boundary conditions

$$
\theta(0)=0, \text { and } \theta(\infty)=\pi / 2 .
$$

We have yet to fix the value of the parameter $a$ in equation (10). To do that, we follow Ref. [9], and choose $a$ in such a way as to achieve exact self-consistency far from the vortex. Standard theory tells us that the order parameter $\Delta=\lambda\left\langle\psi_{\downarrow} \psi_{\uparrow}\right\rangle$ (where $-\lambda$ is the BCS coupling constant)

is obtained by summing the thermal Green function $\int_{0}^{\beta}\left\langle\psi_{\downarrow}(\tau) \psi_{\uparrow}(0)\right\rangle \mathrm{e}^{i \omega \tau} d \tau$ over all Matsubara frequencies $\omega_{l}=(2 l+1) T / \pi$. At temperature $T=1 / \beta=0$ the sum condenses to an integral over the (imaginary) energy axis, and by expressing the thermal Green function through the function $f(u, E)=\sin \theta(u, E)$ one obtains

$$
\Delta(u) / \Delta_{0}=\int_{0}^{\infty} \sin \theta(u,-i \omega) d \omega / \int_{0}^{\infty} \sin \theta(\infty,-i \omega) d \omega .
$$

The function $\sin \theta(u, E)$ is determined by the Usadel equation at energy $E$ (see Appendix C):

$$
-\theta^{\prime \prime}-\frac{\theta^{\prime}}{u}+\frac{\sin 2 \theta}{2 u^{2}}=\frac{u \cos \theta}{\sqrt{u^{2}+a^{2}}}-i \frac{E}{\Delta_{0}} \sin \theta .
$$

The prescription of Ref. [9] now is to expand the solution around the asymptotic limit

$$
\sin \theta(\infty,-i \omega)=\Delta_{0} / \sqrt{\Delta_{0}^{2}+\omega^{2}}, \quad \cos \theta(\infty,-i \omega)=\omega / \sqrt{\Delta_{0}^{2}+\omega^{2}},
$$

find the correction of order $1 / u^{2}$, insert the corrected solution into equation (11), and equate the result for $\Delta(u)$ with the ansatz $\Delta(u) / \Delta_{0}=u / \sqrt{u^{2}+a^{2}}=1-a^{2} / 2 u^{2}+\ldots$ made above. This leads to a linear condition for $a$ giving $a^{2}=\pi / 2$.

We have solved the differential equation (10) with $a^{2}=\pi / 2$ numerically, using Maple. Following again Ref. [9], we integrated the equation from $u=0$ to $u=\infty$, with the derivative $\theta^{\prime}(0)$ so adjusted as to hit the desired value $\pi / 2$ at infinity. The solution is shown in Figure 1 . In the next section we will see how this solution enters into the expression for the coupling constant of the nonlinear $\sigma$ model.

\section{The one-dimensional nonlinear $\sigma$ model}

We are in the process of deriving a low-energy effective theory describing diffusive quasiparticle motion in a disordered vortex. The final step, which we now embark on, begins by taking the solution of the Usadel equation and perturbing it by fluctuations that vary slowly in space. Recalling $T(r, \varphi, z)=1_{p h} \otimes t(r, \varphi, z)$, we put

$$
Q=T Q_{\text {Usadel }} T^{-1}=g \sigma_{3} \otimes t \Sigma_{3} t^{-1}+f \sigma_{2} \mathrm{e}^{-i \varphi \sigma_{3}} \otimes 1 .
$$




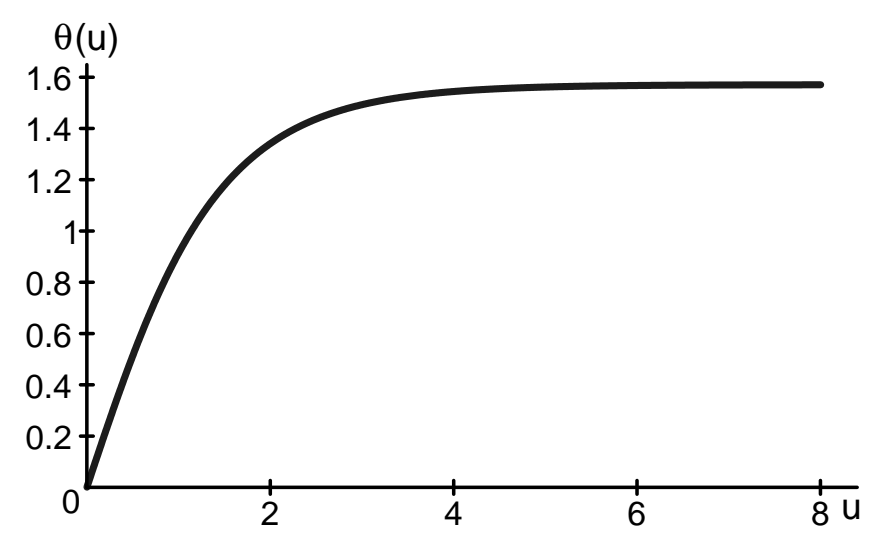

Figure 1: The solution $\theta(u)$ of the differential equation (10) with the boundary conditions $\theta(0)=0$ and $\theta(\infty)=\pi / 2$. The value of the parameter $a^{2}$ was chosen to be $\pi / 2$.

This expression is now inserted into the effective action (5). Let us first evaluate the "kinetic term" STr $\left(\nabla_{A} Q\right)^{2}$. After some calculation we find that the magnetic field disappears completely, the corresponding terms involving either a trace over traceless matrices in the particle-hole space or a supertrace over a supermatrix proportional to the identity. We thus obtain

$$
\operatorname{STr}\left(\nabla_{A} Q\right)^{2}=\mathrm{S} \operatorname{Tr}(\nabla Q)^{2}
$$

The explicit form of the gradient is

$$
\nabla Q=(\nabla g) \sigma_{3} t \Sigma_{3} t^{-1}+g \sigma_{3} \nabla\left(t \Sigma_{3} t^{-1}\right)+\nabla\left(f \sigma_{2} \mathrm{e}^{-i \varphi \sigma_{3}}\right)
$$

By the cyclic invariance of the supertrace and $\mathrm{STr} 1=0$, the kinetic term reduces to

$$
\begin{aligned}
\operatorname{STr}(\nabla Q)^{2} & =g^{2} \operatorname{STr}_{p h \times b f \times c c \times n}\left(1_{p h} \otimes \nabla\left(t \Sigma_{3} t^{-1}\right)\right)^{2} \\
& =2 g^{2} \operatorname{STr}_{b f \times c c \times n}\left(\nabla\left(t \Sigma_{3} t^{-1}\right)\right)^{2} .
\end{aligned}
$$

In the last expression the particle-hole space has disappeared, its only remnant being the factor of 2 multiplying the restricted supertrace. By introducing a reduced $Q$-field,

$$
q=t \Sigma_{3} t^{-1}
$$

we can write more simply

$$
\mathrm{STr}(\nabla Q)^{2}=2 g^{2} \mathrm{~S} \operatorname{Tr}(\nabla q)^{2} .
$$

The other term in $S[Q]$ works out to be

$$
\mathrm{S} \operatorname{Tr}(\tilde{\Delta}+\omega) \sigma_{3} Q=\mathrm{S} \operatorname{Tr} \tilde{\Delta} \sigma_{3} Q_{\text {Usadel }}+g \mathrm{~S} \operatorname{Tr} \omega\left(1_{p h} \otimes t \Sigma_{3} t^{-1}\right)=2 g \mathrm{~S} \operatorname{Tr} \omega q
$$

The effective action for the field $q$ then reads

$$
S[q]=-\frac{\pi \nu}{4} \int d^{3} x \mathrm{STr}\left(g^{2} D(\nabla q)^{2}-4 i g \omega q\right)
$$


To identify the low-energy modes, we use cylindrical coordinates and write the operator controlling the fluctuations around a constant background $q$-field as

$$
-g^{-2} \nabla g^{2} \nabla=-\frac{\partial^{2}}{\partial z^{2}}-\frac{1}{r^{2}} \frac{\partial^{2}}{\partial \varphi^{2}}-\frac{1}{r g^{2}(r)} \frac{\partial}{\partial r}\left(r g^{2}(r) \frac{\partial}{\partial r}\right) .
$$

This operator is self-adjoint in the $L^{2}$-space with measure $g^{2}(r) r d r d \varphi d z$. The spectrum of the first term on the right-hand side is continuous, while the second and third term have a discrete spectrum. [The latter is seen by making a similarity transformation

$$
-\frac{1}{r g^{2}(r)} \frac{\partial}{\partial r}\left(r g^{2}(r) \frac{\partial}{\partial r}\right) \rightarrow-r^{-1 / 2} g^{-1}(r) \frac{\partial}{\partial r} r g^{2}(r) \frac{\partial}{\partial r} r^{-1 / 2} g^{-1}(r)=-\frac{\partial^{2}}{\partial r^{2}}+V(r),
$$

and showing that the effective potential $V(r)$ is confining.] Thus the transverse, i.e. $r$ and $\varphi$, excitations of the $q$-field are gapped (with the gap being of the order of the transverse Thouless energy $D / \xi^{2}$ ), and the relevant low-energy physics is captured by taking $q$ to be a function of $z$ only. Doing so we arrive at the following low-energy effective action for $q=q(z)$ :

$$
S[q]=-\frac{\pi^{2} \nu \xi^{2}}{4} \int d z \operatorname{STr}\left(C_{2} D\left(\partial_{z} q\right)^{2}-4 i C_{1} \omega q\right)
$$

where the dimensionless coefficients $C_{1}$ and $C_{2}$ are given by radial integrals,

$$
C_{n}=2 \int_{0}^{\infty} \cos ^{n} \theta(u) u d u \quad(n=1,2)
$$

which are of order unity. To calculate their precise values one needs the function $\cos \theta(u)$ which is determined by self-consistency. If we take $\theta(u)$ to be the solution of equation (10) with $a^{2}=\pi / 2$, we get $C_{1}=3.16$ and $C_{2}=1.20$. This completes the derivation of the low-energy effective theory. What we have obtained is a one-dimensional field theory that belongs to the general class of nonlinear $\sigma$ models. The expression (12) for its action functional is the first important result of the present paper.

To put the result into context, we elaborate on the definition of the field space. Recall that the field $q(z)$ is a $4 n \times 4 n$ supermatrix $q=t \Sigma_{3} t^{-1}$ with $t(z) \in \operatorname{OSp}(2 n \mid 2 n)$. Clearly, the expression for $q$ is invariant under right multiplication of $t(z)$ by a matrix $h(z)$ that fixes $\Sigma_{3}$ $\left(h \Sigma_{3} h^{-1}=\Sigma_{3}\right)$. By inspection, one easily sees that the group of such matrices is isomorphic to $\mathrm{GL}(n \mid n)$. Thus, the field space of our nonlinear $\sigma$ model is a quotient $\operatorname{OSp}(2 n \mid 2 n) / \mathrm{GL}(n \mid n)$. Let us emphasize that the present theory is distinct from the nonlinear $\sigma$ models that were introduced by Efetov [6] in his work on disordered metallic wires. In fact, what we are dealing with here is one of the new "normal-superconducting" universality classes (namely class $C$ ) identified in Ref. [3]. To demonstrate the distinction clearly, let us take a close-up look at the field space.

Since $\gamma=\left(E_{B B} \otimes \Sigma_{1}-E_{F F} \otimes i \Sigma_{2}\right) \otimes 1_{n}$ gives $\Sigma_{3}=-\gamma\left(\Sigma_{3}\right)^{T} \gamma^{-1}$, the Lie group condition $t=\gamma\left(t^{-1}\right)^{T} \gamma^{-1}$ translates into $q=-\gamma q^{T} \gamma^{-1}$. A useful way of representing $q=t \Sigma_{3} t^{-1}$ is by

$$
q=\left(\begin{array}{cc}
(1+Z \tilde{Z})(1-Z \tilde{Z})^{-1} & -2 Z(1-\tilde{Z} Z)^{-1} \\
2 \tilde{Z}(1-Z \tilde{Z})^{-1} & -(1+\tilde{Z} Z)(1-\tilde{Z} Z)^{-1}
\end{array}\right)
$$

where $Z$ and $\tilde{Z}$ are complex $2 n \times 2 n$ supermatrices. The condition $q=-\gamma q^{T} \gamma^{-1}$ is equivalent to

$$
Z=\left(\begin{array}{cc}
Z^{B B} & Z^{B F} \\
Z^{F B} & Z^{F F}
\end{array}\right)=\left(\begin{array}{cc}
-\left(Z^{B B}\right)^{T} & -\left(Z^{F B}\right)^{T} \\
-\left(Z^{B F}\right)^{T} & \left(Z^{F F}\right)^{T}
\end{array}\right)
$$




$$
\tilde{Z}=\left(\begin{array}{cc}
\tilde{Z}^{B B} & \tilde{Z}^{B F} \\
\tilde{Z}^{F B} & \tilde{Z}^{F F}
\end{array}\right)=\left(\begin{array}{cc}
-\left(\tilde{Z}^{B B}\right)^{T} & \left(\tilde{Z}^{F B}\right)^{T} \\
\left(\tilde{Z}^{B F}\right)^{T} & \left(\tilde{Z}^{F F}\right)^{T}
\end{array}\right)
$$

We see in particular that $Z^{F F}, \tilde{Z}^{F F}$ are symmetric matrices, while $Z^{B B}, \tilde{Z}^{B B}$ are skew. Moreover, stability of the functional integral with action (12) requires

$$
\tilde{Z}^{F F}=-\left(Z^{F F}\right)^{\dagger}, \quad \tilde{Z}^{B B}=+\left(Z^{B B}\right)^{\dagger}, \quad 1-\left(Z^{B B}\right)^{\dagger} Z^{B B}>0 .
$$

When complemented by the $\operatorname{OSp}(2 n \mid 2 n)$ action induced on $Z, \tilde{Z}$ by $q \mapsto t q t^{-1}$, the above properties mean that $Z^{B B}$ and $Z^{F F}$ parametrize certain symmetric domains that are well-known in mathematical physics. Technically speaking, the $B B$-sector is isomorphic to the noncompact symmetric space $\mathrm{SO}^{*}(2 n) / \mathrm{U}(n)$ (called type $D \mathrm{III}$ in Cartan's notation), while the $F F$-sector is isomorphic to the compact symmetric space $\operatorname{Sp}(2 n) / \mathrm{U}(n)$ (called type $C \mathrm{I}$ ). In the terminology of [15], these facts are succinctly summarized by saying that the field space of our nonlinear $\sigma$ model is a Riemannian symmetric superspace of type $D \mathrm{III} \mid C \mathrm{I}$. In contrast, Efetov's models are defined over the Riemannian symmetric superspaces of type $A \mathrm{III}|A \mathrm{III}, B D \mathrm{I}| C \mathrm{II}$, and $C \mathrm{II} \mid B D \mathrm{I}$.

Before turning to the analysis of the one-dimensional field theory, let us briefly discuss its zero-dimensional limit. Such a limit is obtained if the vortex is taken to be isolated, with Neumann boundary conditions $\left(\partial_{z} q\right)(0)=\left(\partial_{z} q\right)(L)=0$ at the two end points $z=0$ and $z=L$. (The boundary conditions on the microscopic quasiparticle wave functions at an insulating boundary are of course Dirichlet, but the reduction to the effective field theory turns them into Neumann.) At small energies $\omega$, the dominant contribution to the functional integral comes from the "zero mode" $q(z)=q_{0}$ (independent of $z$ ). Spatially varying field configurations have a Lagrangian density that scales with the length of the vortex as $L^{-2}$. By comparing terms in the action functional, one sees that they are negligible if

$$
\omega \ll D / L^{2} .
$$

This condition, which delineates the zero-dimensional (or ergodic) regime, is familiar from diffusive metals: ergodic behavior sets in at times much longer than the diffusion time $L^{2} / D$ or, equivalently, at energies much smaller than the Thouless energy $E_{\mathrm{Th}}=D / L^{2}$.

In the zero-dimensional limit, the functional integral over $q(z)$ reduces to a definite integral over the zero mode $q_{0}$ :

$$
\begin{aligned}
\mathcal{Z}[J] & =\int D q_{0} \exp \left(-i \pi \mathrm{S} \operatorname{Tr} \omega q_{0} / 2 \delta+\text { sources }\right) \\
1 / \delta & =2 \pi C_{1} \nu L \xi^{2}
\end{aligned}
$$

To obtain any specific observable, one keeps track of the coupling to the source terms introduced in the starting expression (11). For the special case of the $n$-level correlation function, the corresponding definite integral was computed for all $n$ in [18]. The result is

$$
\langle\nu(E)\rangle=\frac{1}{\delta}-\frac{\sin 2 \pi E / \delta}{2 \pi E}
$$

for the mean density of states as a function of the energy difference $E$ from the Fermi level, and

$$
Y_{2}\left(\varepsilon_{1}, \varepsilon_{2}\right)=\left(\frac{\sin \pi\left(\varepsilon_{1}-\varepsilon_{2}\right)}{\pi\left(\varepsilon_{1}-\varepsilon_{2}\right)}-\frac{\sin \pi\left(\varepsilon_{1}+\varepsilon_{2}\right)}{\pi\left(\varepsilon_{1}+\varepsilon_{2}\right)}\right)^{2} \quad\left(\varepsilon_{i}=E_{i} / \delta\right)
$$

for the two-level cluster function $Y_{2}$. These results agree exactly with the expressions predicted in [3] on the basis of a random matrix hypothesis for systems of symmetry class $C$. 
How does this compare with the results expected on the basis of the traditional approach due to Eilenberger and Usadel? The Usadel equation by itself, i.e. without the nonperturbative extension worked out here, simply yields $1 / \delta$ for the mean density of states at energies comparable to the level spacing. While this answer is correct asymptotically at $E \gg \delta$, it ignores the oscillations seen in (13) and, in particular, it misses the fact that the density of states vanishes at $E=0$. (Note that the Usadel equation predicts secular variations of the density of states over a characteristic scale given by the transverse Thouless energy $D / \xi^{2}$. For our purposes, this is a "high-energy" scale which we shall ignore.)

Why does random matrix theory predict the fine structure of the density of states correctly, whereas the quasiclassical Usadel equation captures only the asymptotics? Perhaps the answer is not entirely obvious and the following remark helpful. What is well understood is that random matrix theory provides a valid description of disordered systems in the ergodic regime, where the physics is governed by the zero modes or constant modes of some field theory. [Recall that the ergodic regime is the region of validity of (13).] One might object, however, that a vortex is a spatially localized object. The solution of the Usadel equation for a disordered vortex is far from being constant but has a characteristic spatial dependence. One might therefore wonder why a zero mode or random matrix argument has any chance of being correct in the present context.

Our analysis has already given the answer to this question: although the solution of the Usadel equation does vary in space, the symmetry group operating on it is independent of space! As we have seen, the random matrix answer is recovered by integrating over the orbit swept out by the (constant) action of the symmetry group. A key feature is the mechanism by which the theory avoids the superficial conflict between constancy of the zero mode and locality of the vortex solution: the orbit generated by the group action contracts to a single point $Q_{\infty}=$ $T Q_{\infty} T^{-1}$ (thereby making the symmetry ineffective) as we move away from the vortex.

To conclude this section, let us mention that similar statements apply also to an SNS junction with phase difference $\phi_{1}-\phi_{2}=\pi$ (symmetry class $C \mathrm{I}$ ). In that case, too, the random matrix predictions of [3] are expected to hold as they stand.

\section{Transmission along the vortex}

Charge transport through a diffusive metallic wire at zero temperature undergoes a crossover from Ohmic behavior to strong localization as the length of the wire is increased. A similar crossover is expected for the transport properties of the normal quasiparticles of a disordered vortex. In the case of metallic wires, the precise form of the crossover functions for the electrical conductance was computed from the solution of Efetov's nonlinear $\sigma$ models in one dimension in [19, 20]. (Note that an error made in these computations for $\beta=4$ was corrected in [21].) We will now embark on a similar project for the vortex, by analysing the one-dimensional field theory found above. Note that for the purpose of testing for localization of normal quasiparticles in a vortex, charge transport is not an experimentally viable option, as the system is short cut by dissipationless charge flow through the superconductor. However, we can imagine probing the transport properties via a measurement of the heat flow along the vortex. Whether such a measurement is experimentally feasible will be discussed in detail in a separate publication.

In this section we will show that the normal quasiparticles in a disordered vortex are subject to a phenomenon called "weak localization", which is well known from disordered metals with time-reversal symmetry and conserved electron spin. Although in normal metals weak localization is destroyed by the application of a magnetic field, in a disordered vortex there exist 
additional modes of quantum interference that arise from Andreev scattering of the quasiparticles and are not suppressed by the presence of a magnetic flux. The zero-dimensional precursor of weak localization in systems of symmetry class $C$ was first noticed in a transfer-matrix calculation by Brouwer and Beenakker [22] and was qualitatively explained in [23].

As a quantitative measure of quasiparticle transport and localization in a disordered vortex, we study the following two-particle Green function:

$$
\tau_{k k^{\prime}}\left(x, x^{\prime} ; E\right)=\sum_{\alpha \alpha^{\prime}}\left\langle v_{k \alpha}^{(x)} G_{\alpha \alpha^{\prime}}^{+}\left(x, x^{\prime} ; E\right) v_{k^{\prime} \alpha^{\prime}}^{\left(x^{\prime}\right)} G_{\alpha^{\prime} \alpha}^{-}\left(x^{\prime}, x ; E\right)\right\rangle,
$$

where $v$ is the left-right symmetric velocity operator

$$
v_{k \alpha}=\frac{1}{2 i m}\left(\left(\sigma_{3}\right)_{\alpha \alpha}\left(\frac{\vec{\partial}}{\partial x^{k}}-\frac{\overleftarrow{\partial}}{\partial x^{k}}\right)-2 i e A_{k}\right)
$$

The superscripts \pm distinguish between retarded and advanced Green functions, and $\alpha, \alpha^{\prime}$ are the particle/hole indices. The angular brackets indicate an average over disorder.

If $\psi=\left\{\psi_{\alpha}\right\}_{\alpha=p, h}$ is a solution of the $\mathrm{BdG}$ equations (with some energy $E$ ), the bilinear $j_{k}(x)=\sum_{\alpha}\left(\bar{\psi}_{\alpha} v_{k \alpha} \psi_{\alpha}\right)(x)$ expresses the $k^{\text {th }}$ component of the probability current carried by a quasiparticle with wavefunction $\psi$. This current is conserved $\left(\sum_{k} \partial_{k} j_{k}=0\right)$, as an immediate consequence of the BdG equations. On the same grounds, the tensor $\tau_{k k^{\prime}}$ satisfies the current conservation laws

$$
\sum_{k} \frac{\partial}{\partial x^{k}} \tau_{k k^{\prime}}\left(x, x^{\prime} ; E\right)=0=\sum_{k^{\prime}} \frac{\partial}{\partial x^{k^{\prime}}} \tau_{k k^{\prime}}\left(x, x^{\prime} ; E\right) .
$$

This suggests that $\tau_{k k^{\prime}}\left(x, x^{\prime} ; E\right)$ can be interpreted as a correlation function of the conserved probability flux carried by quasiparticles with energy $E$.

The nonlocal tensor $\tau_{k k^{\prime}}\left(x, x^{\prime} ; E\right)$ carries more information than we are actually interested in here. To eliminate some of the details, let $P_{z}$ and $P_{z^{\prime}}$ be two planes that intersect the axis of the vortex at right angles, and consider the integrated longitudinal correlation function

$$
\tau(E):=\int_{P_{z}} d^{2} x \int_{P_{z^{\prime}}} d^{2} x^{\prime} \tau_{33}\left(x, x^{\prime} ; E\right) .
$$

By current conservation, this is independent of $z$ and $z^{\prime}$. In fact, $\tau(E)$ is a measure of the "transmittance" of the vortex for quasiparticles of energy $E$, and plays a similar role as the electrical conductance in a metallic wire.

To set up the functional integral for $\tau(E)$, recall the relation

$$
G_{\alpha^{\prime} \alpha}^{-}\left(x^{\prime}, x ; E\right)=-\sum_{\beta \beta^{\prime}} \mathcal{C}_{\alpha \beta} \mathcal{C}_{\alpha^{\prime} \beta^{\prime}} G_{\beta \beta^{\prime}}^{+}\left(x, x^{\prime} ;-E\right)
$$

which follows from the particle-hole symmetry of the BdG Hamiltonian. Introducing two replicas and choosing to represent the Green functions in the bosonic sector, we write

$$
\begin{aligned}
\tau_{k k^{\prime}}\left(x, x^{\prime} ; E\right) & =\sum_{\alpha \alpha^{\prime} \beta \beta^{\prime}} \mathcal{C}_{\alpha \beta} \mathcal{C}_{\alpha^{\prime} \beta^{\prime}}\left\langle\left\langle v_{k \alpha}^{(x)} \psi_{\alpha}^{B 1}(x) \bar{\psi}_{\alpha^{\prime}}^{B 1}\left(x^{\prime}\right) v_{k^{\prime} \alpha^{\prime}}^{\left(x^{\prime}\right)} \psi_{\beta}^{B 2}(x) \bar{\psi}_{\beta^{\prime}}^{B 2}\left(x^{\prime}\right)\right\rangle\right\rangle \\
& =-4 \sum_{\alpha \alpha^{\prime}}\left\langle\left\langle\bar{\Psi}_{\alpha}^{B 22}(x) v_{k \alpha} \Psi_{\alpha}^{B 11}(x) \times \bar{\Psi}_{\alpha^{\prime}}^{B 11}\left(x^{\prime}\right) v_{k^{\prime} \alpha^{\prime}} \Psi_{\alpha^{\prime}}^{B 22}\left(x^{\prime}\right)\right\rangle\right\rangle,
\end{aligned}
$$


where the second line introduces the charge conjugation space, by making use of the relations established in Section 2. The multiple superscript comprises the boson-fermion, replica and charge conjugation indices, in this order. The double angles mean an average over disorder and over the configurations of the fields $\Psi(x)$. The symmetry breaking term in the action functional is given by

$$
\omega=1_{p h} \otimes 1_{b f} \otimes \Sigma_{3} \otimes E \sigma_{3} .
$$

Now, after Hubbard-Stratonovich transformation, integration over the quasiparticle fields and saddle point approximation, we obtain

$$
\tau_{k k^{\prime}}\left(x, x^{\prime} ; E\right)=-\pi^{2} D^{2} \nu^{2}\left\langle\mathcal{J}_{k}^{B 11, B 22}(x) \mathcal{J}_{k^{\prime}}^{B 22, B 11}\left(x^{\prime}\right)\right\rangle,
$$

where

$$
\mathcal{J}=\sum_{\alpha}\left(Q \nabla_{A} Q\right)_{\alpha \alpha} .
$$

For a consistency check on this calculation, note that the Usadel equation yields

$$
\sum_{k} \frac{\partial}{\partial x^{k}} \mathcal{J}_{k}^{B 11, B 22}(x)=0=\sum_{k} \frac{\partial}{\partial x^{k}} \mathcal{J}_{k}^{B 22, B 11}(x),
$$

in agreement with the current conservation law satisfied by the tensor $\tau_{k k^{\prime}}\left(x, x^{\prime} ; E\right)$.

The last step is to use the parametrization of Section 4 for the matrix $Q$ :

$$
Q=g(r) \sigma_{3} \otimes t(z) \Sigma_{3} t^{-1}(z)+f(r) \sigma_{2} \mathrm{e}^{-i \varphi \sigma_{3}},
$$

and to integrate over the planes $P_{z}$ and $P_{z^{\prime}}$. We then obtain

$$
\tau(E)=c_{0}\left\langle j^{B 11, B 22}(z) j^{B 22, B 11}\left(z^{\prime}\right)\right\rangle
$$

where $j=q \partial_{z} q$ and

$$
c_{0}=-4 \pi^{2} D^{2} \nu^{2}\left(\int g^{2}(r) r d r d \varphi\right)^{2}=-\left(2 \pi^{2} C_{2} \nu D \xi^{2}\right)^{2} .
$$

For a diffusive vortex of short length, $L$, we expect the transmittance $\tau(E)$ to obey Ohm's law $(\tau \sim 1 / L)$. Our goal now is to verify this law and compute the weak localization correction, i.e. the next term in an expansion in the ratio of system size over the localization length. To that end, we write the action functional (12) in rescaled form:

$$
S[q]=-\frac{\xi_{\text {loc }}}{16 L} \int_{0}^{1} d s \operatorname{STr}\left(\left(\partial_{s} q\right)^{2}-2 i \epsilon \lambda q\right),
$$

$s$ being the dimensionless coordinate along the vortex. The ratio $L / \xi_{\text {loc }}$, where

$$
\xi_{\text {loc }}=4 \pi^{2} C_{2} \nu D \xi^{2}
$$

will later be identified as a localization length, plays the role of the coupling constant of the one-dimensional field theory. The parameter $\epsilon$,

$$
\epsilon=\frac{2 C_{1} E L^{2}}{C_{2} D},
$$


represents the quasiparticle energy measured in units of the Thouless energy $E_{\mathrm{Th}}=D / L^{2}$, and $\lambda$ is the matrix

$$
\lambda=1_{b f} \otimes \sigma_{3} \otimes \Sigma_{3} .
$$

In keeping with what was said in the introduction, the functional integral is supplemented by open boundary conditions at the two ends of the vortex:

$$
q(0)=\Sigma_{3}=q(1) .
$$

It is well understood that these are the correct boundary conditions to use when the system is in good contact with infinite particle reservoirs. (Roughly speaking, the deviation of $q(z)$ from $\Sigma_{3}$ measures how often a typical Feynman path of the quasiparticle visits the location $z$. A diffusive path that arrives at one of the end points $s=0$ or $s=1$, most likely exits the vortex and disappears into the large phase space of the reservoir, with vanishing probability of return. This draining effect by the coupling to the reservoirs causes the fluctuations of $q$ away from $\Sigma_{3}$ to be suppressed at an open boundary.) On moving the two planes $P_{z}$ and $P_{z^{\prime}}$ to the ends of the vortex $\left(z=0, z^{\prime}=L\right.$ or $\left.s=0, s^{\prime}=1\right)$, the expression for the transmittance $\tau$ becomes

$$
\tau(E)=-\left(\frac{\xi_{\text {loc }}}{2}\right)^{2}\left\langle j^{B 11, B 22}(0) j^{B 22, B 11}(1)\right\rangle \text {. }
$$

\subsection{Parametrization of $q$}

Recall that the supermatrix $q$ satisfies the orthosymplectic condition

$$
q=-\gamma q^{T} \gamma^{-1}, \quad \text { with } \quad \gamma=\left(E_{B B} \otimes \Sigma_{1}-E_{F F} \otimes i \Sigma_{2}\right) \otimes 1_{2} .
$$

The kinetic term $\mathrm{S} \operatorname{Tr}(\partial q)^{2}$ in the action functional $S[q]$ is invariant under global transformations $q(z) \mapsto t q(z) t^{-1}$ with $t \in \mathrm{OSp}(4 \mid 4)$. This symmetry is broken by the potential term $\operatorname{STr} \lambda q$ at finite energy $\epsilon \neq 0$. We will see that increasing the symmetry breaking coupling $\epsilon$ causes crossover to Efetov's nonlinear $\sigma$ model with unitary symmetry (or $\beta=2$ ), thereby eliminating the weak localization correction in the limit $\epsilon \rightarrow \infty$.

The effect of symmetry breaking is best captured by parametrizing $q$ as

$$
q=t_{0} t_{1} \Sigma_{3}\left(t_{0} t_{1}\right)^{-1}
$$

where the matrices $t_{0}, t_{1}$ decompose into blocks in charge conjugation space as follows:

$$
t_{0}=\left(\begin{array}{cc}
1 & Z_{0} \\
\tilde{Z}_{0} & 1
\end{array}\right), \quad t_{1}=\left(\begin{array}{cc}
1 & Z_{1} \\
\tilde{Z}_{1} & 1
\end{array}\right) .
$$

The $4 \times 4$ blocks $Z_{0}, \tilde{Z}_{0}, Z_{1}, \tilde{Z}_{1}$ are chosen to have the following substructure in replica space:

$$
\begin{array}{ll}
Z_{0}=\left(\begin{array}{cc}
0 & z_{0}^{+} \\
z_{0}^{-} & 0
\end{array}\right), & Z_{1}=\left(\begin{array}{cc}
z_{\wedge} & 0 \\
0 & z_{\vee}
\end{array}\right), \\
\tilde{Z}_{0}=\left(\begin{array}{cc}
0 & \tilde{z}_{0}^{+} \\
\tilde{z}_{0}^{-} & 0
\end{array}\right), & \tilde{Z}_{1}=\left(\begin{array}{cc}
\tilde{z}_{\wedge} & 0 \\
0 & \tilde{z}_{\vee}
\end{array}\right) .
\end{array}
$$

Here all quantities $z_{\wedge}, z_{\vee}, z_{0}^{+}, z_{0}^{-}$and their partners with tilde are $2 \times 2$ supermatrices with symmetries that are determined by $q=-\gamma q^{T} \gamma^{-1}$. The rationale behind this choice is that $t_{0}$ commutes with $\lambda\left(t_{0}^{-1} \lambda t_{0}=\lambda\right)$, so that the symmetry breaking term reduces to

$$
\mathrm{S} \operatorname{Tr} \lambda q=\mathrm{S} \operatorname{Tr} \lambda t_{0} t_{1} \Sigma_{3}\left(t_{0} t_{1}\right)^{-1}=\mathrm{S} \operatorname{Tr} \lambda t_{1} \Sigma_{3} t_{1}^{-1},
$$


which simplifies the calculation appreciably. A similar parametrization was used by Altland, Iida and Efetov [14] in their study of the spectral statistics of small metallic particles subject to a symmetry breaking magnetic field. The boundary conditions at $s=0,1$ translate into

$$
Z_{0}(0)=\tilde{Z}_{0}(0)=Z_{0}(1)=\tilde{Z}_{0}(1)=0,
$$

with identical relations in force for $Z_{1}, \tilde{Z}_{1}$. Using these, we can linearize the current $j=q \partial_{z} q$ in $Z_{0}, Z_{1}$ at the boundaries, and the expression for the transmittance becomes

$$
\tau=-\left(\xi_{\text {loc }} / L\right)^{2}\left\langle\partial_{s} z_{0}^{+B B}(0) \partial_{s} \tilde{z}_{0}^{-B B}(1)\right\rangle .
$$

\subsection{Expansion of the kinetic and potential terms}

We will now use standard field theoretic perturbation theory to compute the leading terms of the Taylor expansion in $L / \xi_{\text {loc }}$ for $\tau$. The first step is to expand the action functional $S[q]$ up to fourth order in the fields. Setting $q=t_{0} t_{1} \Sigma_{3}\left(t_{0} t_{1}\right)^{-1}$ we obtain for the kinetic part of the action

$$
\mathrm{S} \operatorname{Tr}\left(\partial_{s} q\right)^{2}=\mathrm{S} \operatorname{Tr}\left[t_{1}^{-1} \partial_{s} t_{1}+t_{1}^{-1}\left(t_{0}^{-1} \partial_{s} t_{0}\right) t_{1}, \Sigma_{3}\right]^{2} .
$$

By inserting the explicit expressions for $t_{0}$ and $t_{1}$ in terms of $Z_{0}, \tilde{Z}_{0}$ etc. and using the fact that $Z_{0}$ and $Z_{1}$ are orthogonal to each other with respect to the supertrace, we get for $\operatorname{STr}\left(\partial_{s} q\right)^{2}$ up to second order

$$
-8 \operatorname{STr}\left(\partial \tilde{Z}_{0} \partial Z_{0}+\partial \tilde{Z}_{1} \partial Z_{1}\right)
$$

and a fourth order contribution given by

$$
\begin{aligned}
& -\quad 8 \operatorname{STr}\left(\partial Z_{0} \partial \tilde{Z}_{0}\left(Z_{1} \tilde{Z}_{1}+Z_{0} \tilde{Z}_{0}\right)+\partial \tilde{Z}_{0} \partial Z_{0}\left(\tilde{Z}_{1} Z_{1}+\tilde{Z}_{0} Z_{0}\right)\right) \\
& +\quad 8 \operatorname{STr}\left(Z_{0} \partial \tilde{Z}_{0}\left(Z_{1} \partial \tilde{Z}_{1}-\partial Z_{1} \tilde{Z}_{1}\right)+\tilde{Z}_{0} \partial Z_{0}\left(\tilde{Z}_{1} \partial Z_{1}-\partial \tilde{Z}_{1} Z_{1}\right)\right) \\
& +\quad 8 \operatorname{STr}\left(\left(\partial Z_{0} \tilde{Z}_{1}\right)^{2}+\left(\partial \tilde{Z}_{0} Z_{1}\right)^{2}-\partial \tilde{Z}_{1} \partial Z_{1} \tilde{Z}_{1} Z_{1}-\partial Z_{1} \partial \tilde{Z}_{1} Z_{1} \tilde{Z}_{1}\right) .
\end{aligned}
$$

For simplicity we have set $\partial \equiv \partial_{s}$. For the potential term we have the expansion

$$
\mathrm{S} \operatorname{Tr} \lambda q=4 \mathrm{~S} \operatorname{Tr}\left(\tilde{z}_{\wedge} z_{\wedge}-\tilde{z}_{\vee} z_{\vee}\right)+4\left(\operatorname{STr}\left(\tilde{z}_{\wedge} z_{\wedge}\right)^{2}-\mathrm{S} \operatorname{Tr}\left(\tilde{z}_{\vee} z_{\vee}\right)^{2}\right)+\ldots
$$

which depends only on the matrix entries of $Z_{1}, \tilde{Z}_{1}$.

\subsection{Propagator and diagrams}

The quadratic part of the field theory action defines the bare propagator. We write

$$
S[q]=-\frac{\xi_{\text {loc }}}{16 L} \int_{0}^{1} d s \mathrm{~S} \operatorname{Tr}\left((\partial q)^{2}-2 i \epsilon \lambda q\right)=S_{2}+S_{4}+\ldots
$$

where

$$
S_{2}=\frac{\xi_{\text {loc }}}{2 L} \int_{0}^{1} d s\left(\mathrm{~S} \operatorname{Tr}(\partial \tilde{Z} \partial Z)+i \epsilon \operatorname{STr}\left(\tilde{z}_{\wedge} z_{\wedge}-\tilde{z}_{\vee} z_{\vee}\right)\right),
$$

and $Z$ in this expression means $Z_{0}+Z_{1}$. From the quadratic part of the action it is clear that the modes of type $Z_{0}$ are massless, while the modes of type $z_{\vee}$ and $z_{\wedge}$ have an imaginary mass term given by $\epsilon$. In the limit $\epsilon \rightarrow \infty$, the latter modes will therefore be completely suppressed. 


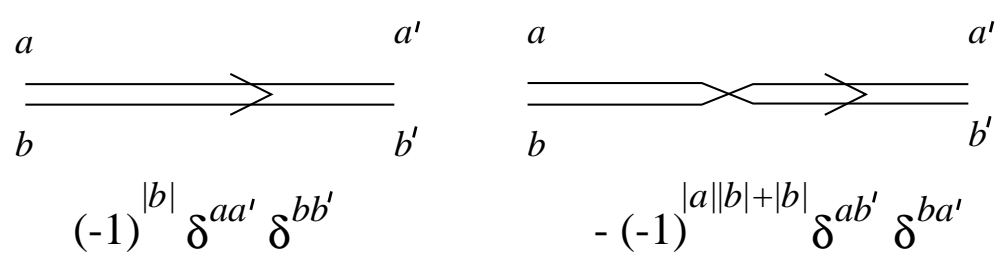

Figure 2: Graphs for the propagator $\left\langle Z^{a b} \tilde{Z}^{b^{\prime} a^{\prime}}\right\rangle$

We have yet to discuss the symmetries of the matrices $Z$ and $\tilde{Z}$. It is easy to see by linearization that the symmetry $q=-\gamma q^{T} \gamma^{-1}$ is equivalent to the condition

$$
X=-\gamma X^{T} \gamma^{-1} \quad \text { on } \quad X=\left(\begin{array}{cc}
0 & Z \\
\tilde{Z} & 0
\end{array}\right) .
$$

On decomposing $\gamma$ into blocks in charge conjugation space,

$$
\gamma=\left(E_{B B} \otimes \Sigma_{1}-E_{F F} \otimes i \Sigma_{2}\right) \otimes 1_{2}=\left(\begin{array}{cc}
0 & \left(\sigma_{3}\right)_{b f} \otimes 1_{2} \\
1_{2} \otimes 1_{2} & 0
\end{array}\right),
$$

we see that the $4 \times 4$ supermatrices $Z=Z_{0}+Z_{1}$ and $\tilde{Z}=\tilde{Z}_{0}+\tilde{Z}_{1}$ must obey

$$
Z=-\sigma Z^{T}, \quad \tilde{Z}=-\tilde{Z}^{T} \sigma
$$

where $\sigma=\left(\sigma_{3}\right)_{b f} \otimes 1_{2}$ is the superparity matrix, with value +1 on bosons and -1 on fermions. Recalling the definition of the supertranspose $T$ and denoting the superparity of $a$ by $|a|$, we equivalently have

$$
Z^{a b}=-(-1)^{|a||b|} Z^{b a}, \quad \tilde{Z}^{a b}=-(-1)^{|a|+|b|+|a||b|} \tilde{Z}^{b a} .
$$

In this notation, the kinetic term reads $\operatorname{STr} \partial \tilde{Z} \partial Z=\sum_{a b}(-1)^{|a|} \partial \tilde{Z}^{a b} \partial Z^{b a}$.

The basic Gaussian integral governing the perturbation expansion is given by

$$
\begin{aligned}
\left\langle Z^{a b}(s) \tilde{Z}^{b^{\prime} a^{\prime}}\left(s^{\prime}\right)\right\rangle_{0} & :=\int \mathcal{D} Z \mathcal{D} \tilde{Z} Z^{a b}(s) \tilde{Z}^{b^{\prime} a^{\prime}}\left(s^{\prime}\right) \exp \left(\frac{\xi_{\text {loc }}}{2 L} \int_{0}^{1} d s \sum_{c d}(-1)^{|c|} \tilde{Z}_{c d} \partial^{2} Z_{d c}\right) \\
& =(-1)^{|b|}\left(\delta^{a a^{\prime}} \delta^{b b^{\prime}}-(-1)^{|a||b|} \delta^{a b^{\prime}} \delta^{b a^{\prime}}\right)\left(L / \xi_{\text {loc }}\right)\left(-\partial^{2}\right)^{-1}\left(s, s^{\prime}\right) .
\end{aligned}
$$

This formula applies to the massless modes $Z_{0}$. For the modes $Z_{1}$ (i.e. $z_{\wedge}$ and $z_{\vee}$ ) one needs to replace $-\partial^{2}$ by $-\partial^{2}+i \epsilon$ and $-\partial^{2}-i \epsilon$ respectively. The index structure on the right-hand side is determined by the super skewsymmetry of the matrices $Z$ and $\tilde{Z}$. It is seen that there are two different ways of propagating the matrix indices, which we represent graphically as shown in Figure 2.

The space dependence of the propagator is given by

$$
\mathrm{D}_{0}\left(s, s^{\prime}\right):=\left(-\partial^{2}\right)^{-1}\left(s, s^{\prime}\right)= \begin{cases}s\left(1-s^{\prime}\right) & \text { for } s \leq s^{\prime} \\ s^{\prime}(1-s) & \text { for } s \geq s^{\prime}\end{cases}
$$

for the massless modes, and by

$$
\begin{aligned}
\mathrm{D}_{\wedge}\left(s, s^{\prime}\right) & :=\left(-\partial^{2}+i \epsilon\right)^{-1}\left(s, s^{\prime}\right) \\
& =\frac{1}{\sqrt{i \epsilon} \sinh \sqrt{i \epsilon}} \begin{cases}\sinh \sqrt{i \epsilon} s \sinh \sqrt{i \epsilon}\left(1-s^{\prime}\right) & \text { for } s \leq s^{\prime}, \\
\sinh \sqrt{i \epsilon} s^{\prime} \sinh \sqrt{i \epsilon}(1-s) & \text { for } s \geq s^{\prime},\end{cases}
\end{aligned}
$$


for those with mass squared equal to $i \epsilon$. Note that these Green functions satisfy Dirichlet boundary conditions at the ends $s=0$ and $s=1$, as required. The Green function $\mathrm{D}_{\vee}\left(s, s^{\prime}\right)=$ $\left(-\partial^{2}-i \epsilon\right)^{-1}\left(s, s^{\prime}\right)$ is obtained from $\mathrm{D}_{\wedge}\left(s, s^{\prime}\right)$ by the substitution $\epsilon \rightarrow-\epsilon$.

We are now ready to calculate the transmittance of the vortex up to one-loop order. What we want is the quantity

$$
\tau=-\left(\xi_{\text {loc }} / L\right)^{2}\left\langle\partial Z_{0}^{B 1, B 2}(0) \partial \tilde{Z}_{0}^{B 2, B 1}(1) \times\left(1-S_{4}\right)\right\rangle_{0},
$$

where $S_{4}$ is the part of the action functional that is quartic in $Z$ and $\tilde{Z}$, and the vacuum expectation value is taken with respect to the Gaussian action $S_{2}$. $S_{4}$ contains terms from the kinetic and the potential part in $S$.

Let us first compute the leading contribution (tree level). Using the expression for the massless propagator we obtain

$$
\begin{aligned}
\tau_{0} & =-\left(\xi_{\text {loc }} / L\right)^{2}\left\langle\partial Z_{0}^{B 1, B 2}(0) \partial \tilde{Z}_{0}^{B 2, B 1}(1)\right\rangle_{0} \\
& =-\left.\left(\xi_{\text {loc }} / L\right) \partial_{s} \partial_{s^{\prime}} \mathrm{D}_{0}\left(s, s^{\prime}\right)\right|_{s=0, s^{\prime}=1}=\xi_{\text {loc }} / L .
\end{aligned}
$$

This is the equivalent of Ohm's law for the electrical conductance. Note that $\partial_{s} \partial_{s^{\prime}} \mathrm{D}_{0}\left(s, s^{\prime}\right)$ is in fact independent of $s$ and $s^{\prime}$, as is expected from conservation of the quasiparticle probability flux. The Ohmic limit $\tau_{0}=\xi_{\text {loc }} / L$ gives support to the interpretation of $\xi_{\text {loc }}$ as a localization length. In a diffusive wire one would have $\xi_{\text {loc }} \sim N \ell$ with $N$ the number of channels open at the Fermi energy or, equivalently, $\xi_{\text {loc }} \sim \nu \mathcal{A} D$ with $\mathcal{A}$ the cross sectional area of the wire. In the present case we saw that

$$
\xi_{\text {loc }} \sim \nu \xi^{2} D
$$

This means that the role of the cross sectional area is here taken by $\xi^{2} \sim D / \Delta_{0} \sim \xi_{0} \ell$.

We are now going to compute the weak localization correction, which is of order $\left(L / \xi_{\text {loc }}\right)^{0}$. First, we notice that the "external legs" $\partial Z_{0}^{B 1, B 2}(0)$ and $\partial \tilde{Z}_{0}^{B 2, B 1}(1)$ can only couple to terms containing at least one $Z_{0}$ and one $\tilde{Z}_{0}$. Therefore the fourth order terms coming from the potential, as well as the terms on the third line in (14), can be omitted from $S_{4}$ for present purposes. In Appendix $\mathrm{E}$ we show that the contributions originating from the terms on the second line of (14) cancel each other, so we need only consider the terms coming from the first line:

$$
\tau_{1}=\frac{\xi_{\text {loc }}^{3}}{2 L^{3}}\left\langle\partial Z_{0}^{B 1, B 2}(0) \partial \tilde{Z}_{0}^{B 2, B 1}(1) \int_{0}^{1} d s \operatorname{STr}\left(Z \tilde{Z} \partial Z_{0} \partial \tilde{Z}_{0}+\tilde{Z} Z \partial \tilde{Z}_{0} \partial Z_{0}\right)\right\rangle,
$$

where we again used the notation $Z=Z_{0}+Z_{1}$ and the fact that $Z_{0}$ and $Z_{1}$ are orthogonal with respect to the supertrace.

Next, we draw the one-loop diagrams that potentially contribute to the transmittance. The ones associated with the term $\operatorname{STr} Z \tilde{Z} \partial Z_{0} \partial \tilde{Z}_{0}$ are displayed in Figure 3. Those associated with STr $\tilde{Z} Z \partial \tilde{Z}_{0} \partial Z_{0}$ are similar, except that the loop is placed in the lower part of the graph. The first two diagrams, which contain a freely running index, vanish by the usual mechanism of supersymmetry: the fermion loops come with a minus sign relative to the boson loops.

Let us now compute the contribution from Diagram 3. In the course of this computation it will become clear that the diagrams with massless loops (type $Z_{0}$ ) are zero. First we consider the following contraction of the fields:

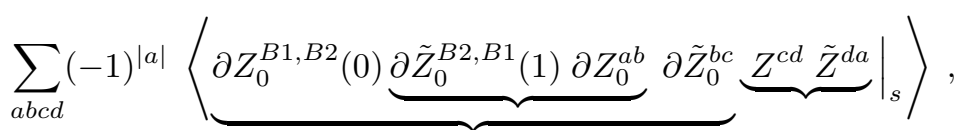


1)

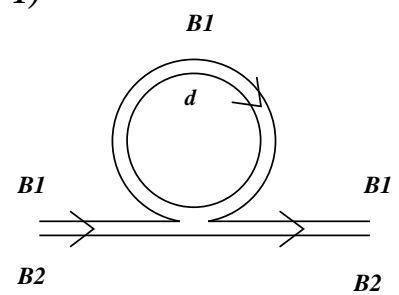

3)

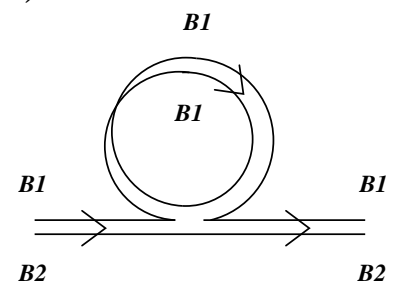

2)

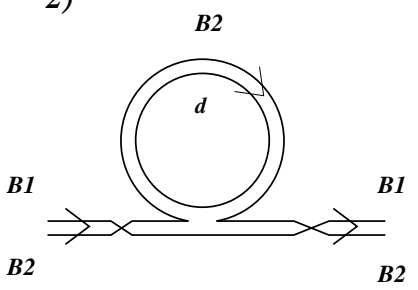

4)

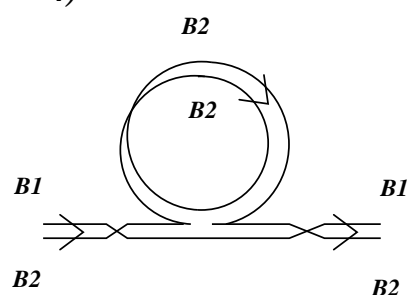

Figure 3: One-loop diagrams potentially contributing to the transmittance.

which yields the expression

$$
\sum_{d}\left\langle\partial Z_{0}^{B 1, B 2}(0) \partial \tilde{Z}_{0}^{B 2, B 1}(s)\right\rangle_{0}\left\langle\partial Z_{0}^{B 1, B 2}(s) \partial \tilde{Z}_{0}^{B 2, B 1}(1)\right\rangle_{0}\left\langle Z^{B 1, d}(s) \tilde{Z}^{d, B 1}(s)\right\rangle_{0} .
$$

The propagator associated with the loop, $\left\langle Z^{B 1, d}(s) \tilde{Z}^{d, B 1}(s)\right\rangle_{0}$, consists of two terms, one containing a factor $(-1)^{|d|} \delta^{B 1, B 1} \delta^{d d}$ (Diagram 1) and another one with $-\delta^{B 1, d} \delta^{d, B 1}$ (Diagram 3). The first term vanishes by supersymmetric cancellation, $\sum_{d}(-1)^{|d|}=0$, as stated above. (Note in particular that all contributions from $Z_{0}$-loops cancel by this mechanism.) The second gives

$$
-\left.\left.\partial_{s_{1}}\right|_{s_{1}=0} \partial_{s} \mathrm{D}_{0}\left(s_{1}, s\right) \mathrm{D}_{\wedge}(s, s) \partial_{s} \partial_{s_{2}}\right|_{s_{2}=1} \mathrm{D}_{0}\left(s, s_{2}\right) .
$$

Diagram 4 gives a very similar contribution, the only difference being that $D_{\vee}(s, s)$ replaces $D_{\wedge}(s, s)$. It should also be clear now that if the external legs are contracted with the fields $Z$ and $\tilde{Z}$, the loop is of $Z_{0}$ type and yields a vanishing contribution.

The same result is obtained when the diagrams arising from $\mathrm{S} \operatorname{Tr} \tilde{\partial} Z_{0} \partial Z_{0} \tilde{Z} Z$ are considered. Although there might in principle be extra contributions from the expansion of the functional integration measure, we show in Appendix $\mathrm{F}$ that this is not the case.

In summary, we obtain the following result for the weak localization correction to the transmittance:

$$
\begin{aligned}
\tau_{1} & =-\left.\left.\int_{0}^{1} d s \partial_{s_{1}} \partial_{s} \mathrm{D}_{0}\left(s_{1}, s\right)\right|_{s_{1}=0}\left(\mathrm{D}_{\wedge}(s, s)+\mathrm{D}_{\vee}(s, s)\right) \partial_{s} \partial_{s_{2}} \mathrm{D}_{0}\left(s, s_{2}\right)\right|_{s_{2}=1} \\
& =-\int_{0}^{1} d s\left(\frac{\sinh \sqrt{i \epsilon} s \sinh \sqrt{i \epsilon}(1-s)}{\sqrt{i \epsilon} \sinh \sqrt{i \epsilon}}+\frac{\sinh \sqrt{-i \epsilon} s \sinh \sqrt{-i \epsilon}(1-s)}{\sqrt{-i \epsilon} \sinh \sqrt{-i \epsilon}}\right) \\
& =-\frac{1}{2}\left(\frac{\operatorname{coth} \sqrt{i \epsilon}}{\sqrt{i \epsilon}}+\frac{\operatorname{coth} \sqrt{-i \epsilon}}{\sqrt{-i \epsilon}}\right) .
\end{aligned}
$$


Using the expansion of the hyperbolic cotangent around $x=0$,

$$
\operatorname{coth} x=\frac{1}{x}+\frac{x}{3}+\ldots,
$$

we find that the weak localization correction for quasiparticles of zero energy is $\tau_{1}(\epsilon=0)=-1 / 3$. In the limit $\epsilon \rightarrow \infty$ the correction vanishes as $1 / \sqrt{\epsilon}$. The crossover between the two regimes takes place at $\epsilon \sim 1$, corresponding to energies of the order of the Thouless energy $E_{\mathrm{Th}}=D / L^{2}$.

To finish this section, let us briefly recall the physical picture [3] explaining the origin of the weak localization correction computed above. Weak localization in normal-/superconducting systems with a magnetic field arises from modes of interference that are present already in the single-particle Green function. The relevant diffusive processes are described by singleparticle Feynman paths containing a closed loop which is traversed twice. (This is the physical interpretation of the third and fourth diagram in Figure 3.) To make such a process phase coherent in the presence of a magnetic field, the charge state of the quasiparticle during the second traversal must be opposite to its state during the first traversal (the charge is reversed by Andreev reflection). In that case, the total magnetic phase accumulated in the loop vanishes, and the process survives disorder averaging. The net effect of such processes is to suppress the density of states and reduce the transmittance of the vortex (weak localization).

The preceding discussion assumes the energy of the quasiparticle to be zero or negligibly small. At finite energy $E$, the phase coherence between the two charge states in the closed loop is reduced by an additional dynamical phase, which is proportional to $E$ multiplied by the time typically spent in the loop. For diffusive dynamics, this time is estimated to be of the order of $L^{2} / D$ in an open system of size $L$. Therefore, phase decoherence due to $E>0$ becomes effective at energies of the order of the Thouless energy $D / L^{2}$.

In the limit $E \gg D / L^{2}$, states of opposite charge can no longer interfere, so the problem reduces to considering BdG particles only, or BdG holes only. The surviving modes of interference are the same as those of a metallic wire subject to a magnetic field. It is well known that the latter system has a vanishing weak localization correction, and one must go to two-loop order to see the onset of localization. The field theory describing such systems is Efetov's nonlinear $\sigma$ model with unitary symmetry $(\beta=2)$. What one expects, then, is that the field space of $q(z)$ becomes isomorphic to Efetov's model space $(\beta=2)$ when the modes $Z_{1}$, which are killed by the limit $E \rightarrow \infty$, are set to zero. Such an isomorphism in fact exists, and will be constructed in the next section.

\section{Strong localization}

To introduce the subject of this section, we briefly recall how the conductance of a disordered quasi-one-dimensional normal metal varies with the length $L$ of the system [24]. Provided that $L$ is not too small, the disorder averaged conductance $g(L)$ depends only on a single scaling variable:

$$
g(L)=F_{\beta}\left(L / \xi_{\text {loc }}\right),
$$

where $\xi_{\text {loc }}$ is a characteristic scale for the onset of localization. The scaling function $F_{\beta}$ is specific to each Wigner-Dyson universality class (with symmetry index $\beta=1,2$, or 4 ). Let the normalization conventions for $\xi_{\text {loc }}$ be such that $F_{\beta}$ has the Ohmic (or small $x$ ) limit $F_{\beta}(x)=$ $1 / x+\ldots$ in all cases. Then, to next order in the small- $x$ expansion,

$$
F_{\beta}(x)=\frac{1}{x}-c_{\beta}+\mathcal{O}(x)
$$


where the constant $c_{\beta}$, the weak localization correction, is $c_{\beta}=2 / 3,0,-1 / 3$ for $\beta=1,2$, 4. Looking back at the result of the previous section, we see that weak localization for the low-energy quasiparticles of a disordered vortex is qualitatively the same as for metallic wires with symmetry index $\beta=1$.

In the opposite limit of large $x$ the scaling functions fall off exponentially:

$$
F_{\beta}(x) \stackrel{x \rightarrow \infty}{\longrightarrow} A_{\beta} \exp (-x / \beta),
$$

which is an expression of the phenomenon of strong localization that occurs in quasi-onedimensional systems. Similar behavior is expected for the transmittance $\tau$ of the vortex. The purpose of the present section is to confirm this expectation and work out the precise value of the exponent governing the exponential decay law.

Recall the formula expressing $\tau$ as a functional integral over fields $Z$ :

$$
\tau=-\left(\xi_{\mathrm{loc}} / L\right)^{2}\left\langle\partial_{s} Z_{0}^{B 1, B 2}(0) \partial_{s} \tilde{Z}_{0}^{B 2, B 1}(1)\right\rangle .
$$

For the purpose of extracting the large- $L$ asymptotics, it is best to abandon the functional integral formulation and pass to an equivalent quantum mechanical description. To do that we interpret the dimensionless coordinate $s$ as an imaginary time. Since the Lagrangian of our system is

$$
\left(\xi_{\text {loc }} / 16 L\right) \mathrm{S} \operatorname{Tr}\left(-\left(\partial_{s} q\right)^{2}+2 i \epsilon \lambda q\right),
$$

the evolution with "time" (i.e. length) will be governed by the Hamiltonian

$$
\mathcal{H}=-\frac{L}{2 \xi_{\text {loc }}} \triangle+\frac{i \epsilon \xi_{\text {loc }}}{8 L} \operatorname{STr} \lambda q,
$$

where $\triangle$ is the Laplace-Beltrami operator of the field space $G_{2} / H_{2}=\operatorname{OSp}(4 \mid 4) / \operatorname{GL}(2 \mid 2)$ with metric tensor $-\mathrm{S} \operatorname{Tr}(\mathrm{d} q)^{2} / 8$. The open boundary conditions on the fields mean that we want a quantum transition amplitude from $Z=0$ at $s=0$ to $Z=0$ at $s=1$. Moreover, by the rule that $\dot{x}$ for a Euclidean path integral with Lagrangian density $m \dot{x}^{2} / 2+V(x)$ translates into the differential operator $m^{-1} d / d x$ in the quantum theory, the expression for $\tau$ becomes

$$
\tau=\left\langle Z=0\left|D_{0}^{B 1, B 2} \mathrm{e}^{-\mathcal{H}} \tilde{D}_{0}^{B 2, B 1}\right| Z=0\right\rangle,
$$

where $D_{0}^{B 1, B 2}=\partial / \partial \tilde{Z}_{0}^{B 2, B 1}$ and $\tilde{D}_{0}^{B 2, B 1}=\partial / \partial Z_{0}^{B 1, B 2}$.

Supersymmetry guarantees the ground state of $\mathcal{H}$ to be exactly at zero. By inserting a complete set of eigenstates of $\mathcal{H}$, we see that the exponential decay of $\tau$ for large $L$ is determined by the smallest eigenvalue of $\mathcal{H}$ such that the corresponding eigenfunction $\psi$ has nonzero matrix element $\left\langle 0\left|D_{0}^{B 1, B 2}\right| \psi\right\rangle$. Unfortunately, computing the spectrum (or even the lowest nonzero eigenvalue) of $\mathcal{H}$ for all $\epsilon$ is a rather difficult task. The only cases we can do easily are $\epsilon=0$ and $\epsilon=\infty$. These are the cases we will restrict ourselves to here.

Consider the case $\epsilon=0$ first. The Hamiltonian we are facing is minus the Laplacian on $G_{2} / H_{2}$. Because the Laplacian commutes with the action of the symmetry group $G_{2}$, its spectrum is in principle computable by the powerful machinery of Lie group theory. There exists, however, a trick we can use to further simplify the problem. Although we are forced to introduce two replicas to average a product of one retarded and one advanced Green function when the quasiparticle energy $E$ is nonzero, in the limit $E=0=\epsilon$ we can make do with just a single replica. The idea is to use again the BdG particle-hole symmetry to express $G^{-}(E)$ by $G^{+}(-E)$, 
and then to represent one Green function in the bosonic sector and the other one in the fermionic sector. This can be done without breaking the supersymmetry of the formalism if and only if $E=0$. Developing the rest of the theory in the same manner as before, we arrive at a reduced description in terms of a "small" $q$ field taking values in $G_{1} / H_{1}=\operatorname{OSp}(2 \mid 2) / \mathrm{GL}(1 \mid 1)$. (The external derivatives $D_{0}^{B 1, B 2}$ and $\tilde{D}_{0}^{B 2, B 1}$ in the transition amplitude formula for $\tau$ are replaced by odd derivatives $D^{B F}$ and $\tilde{D}^{F B}$.)

To obtain the lowest nonzero eigenvalue of minus the Laplacian on $G_{1} / H_{1}$ with a minimal amount of mathematical effort, we adopt the pedestrian approach of choosing a suitable coordinate system and expressing the Laplacian as a differential operator in those specific coordinates. A particularly useful coordinate system is provided by the following polar decomposition:

$$
\begin{aligned}
q & =h a \Sigma_{3} a^{-1} h^{-1}, \quad a=\exp \left(\begin{array}{cc}
0 & Y \\
Y & 0
\end{array}\right), \quad Y=\left(\begin{array}{cc}
0 & 0 \\
0 & i \theta
\end{array}\right), \\
h & =\exp \left(\begin{array}{cc}
h_{1}^{T} & 0 \\
0 & h_{1}^{-1}
\end{array}\right), \quad h_{1}=\exp \left(\begin{array}{cc}
0 & \zeta \\
\bar{\zeta} & 0
\end{array}\right) \exp \left(\begin{array}{cc}
0 & 0 \\
0 & i \varphi
\end{array}\right) .
\end{aligned}
$$

Here $\theta, \varphi$ are real commuting variables, and $\zeta, \bar{\zeta}$ are complex anticommuting. We refer to $\theta$ as the "radial" coordinate. The metric tensor in this coordinate system takes the form

$$
-\mathrm{S} \operatorname{Tr}(\mathrm{d} q)^{2} / 8=\mathrm{d} \theta^{2}+\sin ^{2} 2 \theta(\mathrm{d} \varphi+\bar{\zeta} \mathrm{d} \zeta / 2 i+\zeta \mathrm{d} \bar{\zeta} / 2 i)^{2}+\sin ^{2} \theta \mathrm{d} \zeta \mathrm{d} \bar{\zeta}
$$

Now, there exists a standard formula for the Laplacian on a Riemannian (super)manifold:

$$
\triangle=\sum_{i j}(-1)^{|i|} g^{-1 / 2}(\xi) \partial_{\xi^{i}} g^{i j}(\xi) g^{1 / 2}(\xi) \partial_{\xi^{j}}
$$

if the metric tensor is $\sum \mathrm{d} \xi^{i} g_{i j}(\xi) \mathrm{d} \xi^{j}$, and $g(\xi)=\operatorname{SDet}\left(g_{i j}(\xi)\right)$. Given this formula one can write down a lengthy expression for the Laplacian on $G_{1} / H_{1}$. However, there is yet another simplification and we do not need to go through the trouble of writing the full expression. The point is that we can construct the desired eigenfunction of $\triangle$ by repeatedly acting with symmetry generators on a corresponding radial eigenfunction $f(\theta)$. It is therefore sufficient to study the spectrum of the radial Laplacian, which from the above expression for the metric is

$$
\triangle^{\#}=\left(\frac{\sin 2 \theta}{\sin ^{2} \theta}\right)^{-1} \frac{d}{d \theta}\left(\frac{\sin 2 \theta}{\sin ^{2} \theta}\right) \frac{d}{d \theta} .
$$

The low lying spectrum of this operator can be constructed very simply by trial and error. Making the substitution $x=\cos 2 \theta$ we have

$$
-\triangle^{\#} / 4=-(1-x) \frac{d}{d x}(1+x) \frac{d}{d x}
$$

and it is easy to verify that this has the eigenfunctions

$$
f_{0}=1, \quad f_{1}=1-x, \quad f_{2}=(1-x)(1+3 x)
$$

with eigenvalues $\lambda_{0}=0, \lambda_{1}=1$, and $\lambda_{2}=4$. The pattern we see suggests that the complete spectrum of $-\triangle^{\#} / 4$ might be $n^{2}$ with $n$ an integer. (This guess is in fact confirmed by more informed Lie algebraic considerations.) In any case, it is clear that the lowest nonzero eigenvalue is $\lambda_{1}=1$. By applying to $f_{1}=1-x$ an odd generator of the symmetry group $G_{1}$, 
one can explicitly construct an odd eigenfunction $\psi$ of $-\triangle / 4$ with eigenvalue +1 and matrix element $\left\langle 0\left|D^{B F}\right| \psi\right\rangle \neq 0$. Since the Hamiltonian is $\mathcal{H}=-L \triangle / 2 \xi_{\text {loc }}$, we predict the decay of the transmittance to be

$$
\tau(E=0) \sim \exp \left(-2 L / \xi_{\text {loc }}\right)
$$

for quasiparticles with zero energy.

The other limit $E \rightarrow \infty$ can be done more quickly, by drawing on known results. As we saw, this limit eliminates the modes $Z_{1}$, and what remains are the modes $Z_{0}$, which are recalled to have the matrix representation

$$
Z_{0}=\left(\begin{array}{cc}
0 & z_{0}^{+} \\
z_{0}^{-} & 0
\end{array}\right), \quad \tilde{Z}_{0}=\left(\begin{array}{cc}
0 & \tilde{z}_{0}^{+} \\
\tilde{z}_{0}^{-} & 0
\end{array}\right)
$$

By the off-diagonal structure of these matrices, the field space decomposes into two separate pieces, which are isomorphic copies of each other by the orthosymplectic relations $Z_{0}=-\sigma Z_{0}^{T}$ and $\tilde{Z}_{0}=-\tilde{Z}_{0}^{T} \sigma$. We can write the Lagrangian in terms of one of these copies, say $z_{0}^{+}$ and its partner $\tilde{z}_{0}^{-}$, and account for the other one by multiplying by 2 . The kinetic term $-\left.\left(\xi_{\text {loc }} / 16 L\right) \operatorname{STr}(\partial q)^{2}\right|_{Z_{1}=0}$ then takes the form

$$
\left(\xi_{\text {loc }} / L\right) \operatorname{STr}\left(1-\tilde{z}_{0}^{-} z_{0}^{+}\right)^{-1} \partial \tilde{z}_{0}^{-}\left(1-z_{0}^{+} \tilde{z}_{0}^{-}\right)^{-1} \partial z_{0}^{+} .
$$

Recall that $z_{0}^{+}$and $\tilde{z}_{0}^{-}$are complex $2 \times 2$ supermatrices unconstrained by any symmetry relations that do not involve complex conjugation. This means that the object at hand is the Lagrangian of Efetov's nonlinear $\sigma$ model for metallic wires of the Wigner-Dyson symmetry class $\beta=2$, in the specific parametrization given by

$$
Q=\left(\begin{array}{cc}
1 & z_{0}^{+} \\
z_{0}^{-} & 1
\end{array}\right)\left(\begin{array}{cc}
1 & 0 \\
0 & -1
\end{array}\right)\left(\begin{array}{cc}
1 & z_{0}^{+} \\
\tilde{z}_{0}^{-} & 1
\end{array}\right)^{-1}
$$

and with a renormalized value of the localization length. This model has been much studied in the literature $[25,19,20]$. Taking the result from there, we find

$$
\tau(E=\infty) \sim \exp \left(-L / 4 \xi_{\text {loc }}\right) .
$$

Thus the localization length at $\epsilon=0$ is predicted to be shorter than at $\epsilon \gg 1$ by a large factor of 8. This strong energy dependence already announced itself in the weak localization correction, and now we see that it becomes even more dramatic in the region of strong localization.

\section{Concluding remarks}

In this paper we have established a field theoretic description of the diffusive motion of normal quasiparticles along the core of a vortex in a dirty, extreme type-II, s-wave superconductor. The technique used was an extension, due to Ref. [11], of the supersymmetric method developed by Efetov and others for the purpose of studying localization and mesoscopic phenomena in normal metals. A comprehensive account of this extension and its application to normal/superconducting mesoscopic systems will appear shortly [26].

In a simplified picture, one may view a vortex core as a normal conducting wire surrounded by superconducting walls. The quasiparticles in such a system undergo Andreev scattering and partake in interference processes that are not present in a disordered normal metal. This gives rise to different spectral correlations and transport properties. For a short, isolated vortex 
the correlations of the quasiparticle spectrum (in the universal low-energy limit) have been verified to agree with those of a random matrix of symmetry class $C$. For an open system and quasiparticle energies below $E_{\mathrm{Th}}=D / L^{2}$, the "transmittance" defined in Section 同 exhibits a weak localization correction. Strong localization sets in for vortices of length $L>\xi_{\text {loc }}$. The localization length $\xi_{\text {loc }}$ for a quasiparticle with energy above the Thouless energy $E_{\mathrm{Th}}$ is eight times larger than the localization length of a low-energy quasiparticle.

These results were obtained by reducing the effective field theory for the quasiparticles to a one-dimensional nonlinear $\sigma$ model. In the process, we had to solve a saddle point equation, which turned out to be the Usadel equation. The saddle point approximation is valid if $\mu \gg 1 / \tau$ and $1 / \tau \gg \Delta_{0}$, the latter being known as the dirty limit. The reduction to one dimension (the degree of freedom along the vortex) can be done for quasiparticle energies smaller than the transverse Thouless energy $D / \xi^{2}$.

It is a well known fact that nonmagnetic, isotropic disorder has no significant effect on s-wave superconductivity (Anderson's theorem). This ceases to be true when the order parameter is not spatially homogeneous. Then, a self-consistent field treatment is required. The self-consistency problem for the disordered vortex has been studied both in the framework of Landau-Ginzburg theory [13], and by using the Usadel equation [10. Both approaches show that disorder with an elastic mean-free path $\ell$ changes the coherence length from its clean value $\xi_{0}$ to a dirty value $\xi \sim\left(\ell \xi_{0}\right)^{1 / 2}$. We have seen that this scaling of the dirty coherence length comes about quite naturally, by using no more than dimensional analysis and some minimal assumptions about the shape of the condensate.

We did not implement an exact self-consistent treatment here. Instead, we took the order parameter profile $\Delta(r) / \Delta_{0}$ from a simple one-parameter family of functions and chose the free parameter so as to achieve self-consistency in the region of approach to the asymptotics far from the vortex. We believe that an exact treatment will lead only to minor corrections in the numerical constants $C_{1}$ and $C_{2}$ that enter the coupling constants of the nonlinear $\sigma$ model. In any event, the qualitative picture that emerges from our results, namely, a crossover between the low-energy regime with weak localization present in the "transmittance" along the vortex, and the high-energy regime without weak localization, is a robust feature that will not be modified by a more quantitative treatment.

For typical values of a superconductor with a normal metallic diffusion coefficient of the order of $20 \mathrm{~cm}^{2} / \mathrm{sec}$, and a critical temperature around $10 \mathrm{~K}$, a rough estimate of the localization length gives a range $1-10 \mathrm{~mm}$. This corresponds to an elastic mean-free path of the order of $5 \mathrm{~nm}$ and a coherence length of about $100 \mathrm{~nm}$. For a sample of $1-10 \mu \mathrm{m}$ thickness, the Thouless energy of the vortex will be in the $\mathrm{mK}$ range. Because of the large localization length, the weak localization correction is a relatively small effect. Still, the predicted weak localization correction to the transmittance along the vortex should be visible as a low-temperature anomaly in the heat conductivity of an ensemble of vortices between two electron reservoirs. We shall discuss this effect in detail in a forthcoming communication.

Note added. After completion of this manuscript, we learned of related work by Skvortsov, Kravtsov, and Feigel'man 27].

Acknowledgment. We are much indebted to Alexander Altland for valuable discussions and input at many stages of the present work, and for locating Ref. [9]. The initial spark for this research came from Gerd Bergmann, who a number of years ago asked one of us (M.R.Z.) how the disordered vortex relates to the conventional classification scheme for disordered electron systems. 


\section{A Decoupling of the slow modes}

Consider the quartic term

$$
\frac{v^{2}}{2} \int d^{3} x\left(\bar{\Psi}(x) \sigma_{3} \Psi(x)\right)^{2}
$$

which appears on averaging over the random potential $V(x)$. We want to isolate in it the collective modes with small momentum, $|q|<q_{0}$, which are to be decoupled by a HubbardStratonovich transformation. To do that, we Fourier transform to the momentum representation:

$$
\int d^{3} x\left(\bar{\Psi}(x) \sigma_{3} \Psi(x)\right)^{2}=\int \frac{d k_{1}}{(2 \pi)^{3}} \frac{d k_{2}}{(2 \pi)^{3}} \frac{d k_{3}}{(2 \pi)^{3}} \bar{\Psi}\left(k_{1}\right) \sigma_{3} \Psi\left(k_{2}\right) \bar{\Psi}\left(k_{3}\right) \sigma_{3} \Psi\left(-k_{1}-k_{2}-k_{3}\right) .
$$

There exist three independent ways of pairing two fast single-particle momenta to form a slow two-particle momentum $q$ :

$$
\begin{array}{ccccc} 
& \bar{\Psi}\left(k_{1}\right) & \Psi\left(k_{2}\right) & \bar{\Psi}\left(k_{3}\right) & \Psi\left(-k_{1}-k_{2}-k_{3}\right) \\
\text { a) } & k & -k+q & k^{\prime} & -k^{\prime}-q \\
b) & k & -k^{\prime}-q & -k+q & k^{\prime} \\
c) & k & k^{\prime} & -k^{\prime}-q & -k+q
\end{array}
$$

Term a) can be decoupled trivially, producing no more than energy shifts that can be absorbed by a redefinition of the chemical potential. The other two terms can be rearranged in the following way. For term $b$ ) we have

$$
\begin{aligned}
& \sum_{k, k^{\prime}, q} \bar{\Psi}(k) \sigma_{3} \Psi\left(-k^{\prime}-q\right) \bar{\Psi}(-k+q) \sigma_{3} \Psi\left(k^{\prime}\right) \\
= & \sum_{k, k^{\prime}, q} \bar{\Psi}(k) \sigma_{3} \Psi\left(-k^{\prime}-q\right) \Psi^{T}\left(k^{\prime}\right) \sigma_{3} \bar{\Psi}^{T}(-k+q) \\
= & \sum_{k, k^{\prime}, q} \bar{\Psi}(k) \sigma_{3} \Psi\left(-k^{\prime}-q\right)\left(-\bar{\Psi}\left(k^{\prime}\right) \gamma^{-1} \mathcal{C}\right) \sigma_{3}\left(\gamma \mathcal{C}^{-1} \Psi(-k+q)\right) \\
= & \sum_{q} \operatorname{STr}\left(\sum_{k^{\prime}} \Psi\left(-k^{\prime}-q\right) \bar{\Psi}\left(k^{\prime}\right) \sigma_{3}\right)\left(\sum_{k} \Psi(-k+q) \bar{\Psi}(k) \sigma_{3}\right) .
\end{aligned}
$$

Here we have used $\mathcal{C} \sigma_{3} \mathcal{C}^{-1}=-\sigma_{3}$ and the relations

$$
\bar{\Psi}^{T}=\gamma \mathcal{C}^{-1} \Psi, \quad \Psi^{T}=-\bar{\Psi} \gamma^{-1} \mathcal{C}
$$

which follow from $\Psi=\mathcal{C} \gamma^{-1} \bar{\Psi}^{T}$ and $\bar{\Psi}=-\Psi^{T} \mathcal{C}^{-1} \gamma$ (see the main text). The term $c$ ) is the "nice" one and is easily put in the very same form, by using only the cyclic invariance of the supertrace. Thus, the quartic interaction $\left(v^{2} / 2\right) \int d^{3} x\left(\bar{\Psi} \sigma_{3} \Psi\right)^{2}$ is replaced by

$$
v^{2} \sum_{|q|<q_{0}} \mathrm{~S} \operatorname{Tr} \zeta(-q) \zeta(q)
$$

where $\zeta$ is given by a sum of dyadic products of the fields $\Psi$ and $\bar{\Psi}$ :

$$
\zeta(q)=\sum_{k} \Psi(-k+q) \bar{\Psi}(k) \sigma_{3}
$$




\section{B Effective action}

We want to extract from the functional $\mathcal{S}$ defined in (3) a "low-energy" effective action for slowly varying fields $Q(x)=T(x) Q_{0} T^{-1}(x)$ generated from the saddle point $Q_{0}=\sigma_{3} \otimes \Sigma_{3}$. To do that, we insert $Q(x)=T(x) Q_{0} T^{-1}(x)$ into $\mathcal{S}$ :

$$
\mathcal{S}\left[T Q_{0} T^{-1}\right]=\frac{1}{2} \int d^{3} x \operatorname{STr} \ln \left(\mathcal{H}_{0}-\omega+\frac{i \sigma_{3}}{2 \tau} T Q_{0} T^{-1}\right)(x, x),
$$

and expand with respect to $\omega, \tilde{\Delta}$, and $A$, and up to two gradients of $Q(x)$, which are assumed to be "small" quantities.

Let us first extract the gradients. For that, we set $\omega, \tilde{\Delta}$ and $A$ to zero and consider

$$
S_{0}=\frac{1}{2} \int d^{3} x \operatorname{STr} \ln \left(\sigma_{3}\left(-\nabla^{2} / 2 m-\mu\right)+\frac{i \sigma_{3}}{2 \tau} T Q_{0} T^{-1}\right)(x, x) .
$$

This cannot readily be expanded as it stands, as $T(x)$ is ambiguous by multiplication on the right by an arbitrary field $h(x)$ with $h(x) Q_{0} h^{-1}(x)=Q_{0}$. To fix the ambiguity, we write $T(x)$ as

$$
T(x)=g \mathrm{e}^{X(x)} \quad \text { where } \quad X(x) Q_{0}+Q_{0} X(x)=0 .
$$

The matrix $g$ is chosen in such a way that $g Q_{0} g^{-1}$ coincides with the local "mean" of $Q(x)$, while $X(x)$ parametrizes small deviations from that mean. The space dependence of $g$ is assumed to be so weak that it can be neglected for the present purposes.

When $Q=g \mathrm{e}^{X} Q_{0} \mathrm{e}^{-X} g^{-1}$ is inserted into $S_{0}$, the constant matrix $g$ drops out by the cyclic invariance of the supertrace, and expansion of the logarithm in powers of the small quantity $X(x)$ gives

$$
\begin{aligned}
S_{0}=\frac{1}{2} \int d^{3} x \operatorname{STr} \ln \left(\frac{-\nabla^{2}}{2 m}\right. & \left.-\mu+\frac{i}{2 \tau} \mathrm{e}^{X} Q_{0} \mathrm{e}^{-X}\right)(x, x) \\
=-\frac{1}{8 \tau^{2}} \int d^{3} x \int d^{3} x^{\prime} \mathrm{S} & \operatorname{Sr}\left(\frac{-\nabla^{2}}{2 m}-\mu+\frac{i}{2 \tau} Q_{0}\right)^{-1}\left(x, x^{\prime}\right)\left(X\left(x^{\prime}\right)-X(x)\right) \\
\times & \left(\frac{-\nabla^{2}}{2 m}-\mu+\frac{i}{2 \tau} Q_{0}\right)^{-1}\left(x^{\prime}, x\right)\left(X(x)-X\left(x^{\prime}\right)\right)+\ldots .
\end{aligned}
$$

We assume the elastic mean-free path $\ell$ to be much larger than the Fermi wave length $\left(k_{F} \ell \gg 1\right)$. The Green functions then are well approximated by

$$
\left(\frac{-\nabla^{2}}{2 m}-\mu \pm \frac{i}{2 \tau}\right)^{-1}\left(x, x^{\prime}\right) \simeq \frac{m}{2 \pi} \frac{\exp \left(\mp i k_{F}\left|x-x^{\prime}\right|\right)}{\left|x-x^{\prime}\right|} \mathrm{e}^{-\left|x-x^{\prime}\right| / 2 \ell},
$$

and Taylor expansion of the fields

$$
X(x)-X\left(x^{\prime}\right)=\sum_{k=1}^{3}\left(x^{k}-x^{\prime k}\right) \frac{\partial X}{\partial x^{k}}+\ldots
$$

leads to the integral

$$
\int d^{3} x^{\prime} \frac{\left|x-x^{\prime}\right|^{2}}{3}\left|\left(\frac{-\nabla^{2}}{2 m}-\mu+\frac{i}{2 \tau}\right)^{-1}\left(x, x^{\prime}\right)\right|^{2}=\frac{2 m^{2} \ell^{3}}{3 \pi} .
$$


Assembling factors we obtain

$$
S_{0}=\frac{m^{2} \ell^{3}}{12 \pi \tau^{2}} \int d^{3} x \operatorname{STr}(\nabla X)^{2} .
$$

By recalling the formulas $\nu=m k_{F} / 2 \pi^{2}=m^{2} \ell / 2 \pi^{2} \tau$ for the local density of states and $D=$ $\ell^{2} / 3 \tau$ for the diffusion constant in three dimensions, we can write this as

$$
S_{0}=\frac{\pi \nu D}{2} \int d^{3} x \operatorname{STr}(\nabla X)^{2} .
$$

To put the result in invariant form, we use

$$
\mathrm{S} \operatorname{Tr}(\nabla Q)^{2}=\mathrm{S} \operatorname{Tr}\left(\nabla\left(g \mathrm{e}^{X} Q_{0} \mathrm{e}^{-X} g^{-1}\right)\right)^{2}=\mathrm{S} \operatorname{Tr}\left(\nabla \mathrm{e}^{2 X}\right)\left(\nabla \mathrm{e}^{-2 X}\right)=-4 \mathrm{~S} \operatorname{Tr}(\nabla X)^{2}+\ldots
$$

This gives

$$
S_{0}=-\frac{\pi \nu D}{8} \int d^{3} x \operatorname{STr}(\nabla Q)^{2} .
$$

which is the kinetic part of the effective action (5).

The dependence of the Lagrangian on the magnetic field is easily restored by the following symmetry argument. Consider a gauge transformation

$$
A \mapsto A+\frac{1}{e} \nabla \varphi, \quad \psi \mapsto \mathrm{e}^{i \varphi \sigma_{3}} \psi
$$

The transformation law induced on the composite field $Q \sim \Psi \bar{\Psi} \sigma_{3}$ is

$$
Q \mapsto \mathrm{e}^{i \varphi \sigma_{3}} Q \mathrm{e}^{-i \varphi \sigma_{3}},
$$

so the gradient $\nabla Q$ transforms as

$$
\nabla Q \mapsto \nabla Q+i(\nabla \varphi)\left[\sigma_{3}, Q\right] .
$$

This determines the covariant derivative $\nabla_{A}$ acting on $Q$ to be

$$
\nabla_{A}=\nabla-i e A\left[\sigma_{3}, \cdot\right] .
$$

Therefore, the unique way of making the effective action gauge invariant is to substitute

$$
\mathrm{S} \operatorname{Tr}(\nabla Q)^{2} \rightarrow \operatorname{STr}\left(\nabla_{A} Q\right)^{2},
$$

which restores the dependence on the magnetic field without any calculation.

Finally, we expand (3) to extract the terms linear in $\tilde{\Delta}$ and $\omega$. These are

$$
S_{1}=\frac{1}{2} \int d^{3} x \operatorname{STr}\left(\frac{-\nabla^{2}}{2 m}-\mu+\frac{i}{2 \tau} Q\right)^{-1}(x, x) \sigma_{3}(\tilde{\Delta}-\omega) .
$$

By using the saddle point equation $\left(-\nabla^{2} / 2 m-\mu+i Q / 2 \tau\right)^{-1}(x, x)=-i \pi \nu Q(x)$ and the relation $\sigma_{3} \tilde{\Delta}=-\tilde{\Delta} \sigma_{3}$ we immediately get

$$
S_{1}=\frac{i \pi \nu}{2} \int d^{3} x \operatorname{STr} Q(\tilde{\Delta}+\omega) \sigma_{3} .
$$

(Note that, since $Q$ and $\tilde{\Delta}$ behave in the same way under gauge transformations, the term $\mathrm{S} \operatorname{Tr} Q \tilde{\Delta} \sigma_{3}$ is gauge invariant, as is $\mathrm{S} \operatorname{Tr} Q \omega \sigma_{3}$.) Summing the terms $S_{0}$ and $S_{1}$ we arrive at the effective action (5). The sign differences compared to [1] come from: a conjugation of $Q$ by $\sigma_{3}$ (which changes the sign of the off-diagonal terms of $Q$ in the particle-hole space), and an overall change of sign of $Q$. 


\section{Differential equation}

To solve the Usadel equation for a vortex configuration, we made the ansatz $Q_{0}=g(r) \sigma_{3} \otimes$ $\Sigma_{3}+f(r) \sigma_{2} \mathrm{e}^{-i \varphi \sigma_{3}} \otimes 1_{c c}$ in Section 3.3. The purpose of this appendix is to derive the differential equation obeyed by the coefficient functions $f$ and $g$.

To begin, we recall the Usadel equation for $Q$ :

$$
D \nabla_{A}\left(Q \nabla_{A} Q\right)=-i\left[Q,(\tilde{\Delta}+\omega) \sigma_{3}\right] .
$$

Since all of the quantities $Q_{0}, A \sigma_{3}, \tilde{\Delta}=|\Delta| \sigma_{1} \exp \left(-i \varphi \sigma_{3}\right)$ and $\omega=E \Sigma_{3}$ are diagonal in charge conjugation space, we may specialize to the sector where $\Sigma_{3}$ equals unity and work with the simpler ansatz

$$
Q=g(r) \sigma_{3}+f(r) \sigma_{2} \mathrm{e}^{-i \varphi \sigma_{3}}
$$

(Alternatively, we may imagine absorbing $\Sigma_{3}$ into $g$.) The right-hand side of the Usadel equation then becomes

$$
-i\left[g \sigma_{3}, \tilde{\Delta} \sigma_{3}\right]-i\left[f \sigma_{2} \mathrm{e}^{-i \varphi \sigma_{3}}, E \sigma_{3}\right]=(i|\Delta| g+E f) 2 \sigma_{1} \mathrm{e}^{-i \varphi \sigma_{3}} .
$$

The evaluation of the left-hand side of the Usadel equation is more laborious. From $\nabla_{A}=$ $\nabla-i e A\left[\sigma_{3}, \cdot\right]$ we have

$$
\nabla_{A}\left(Q \nabla_{A} Q\right)=\nabla(Q \nabla Q)-i e \nabla\left(Q\left[A \sigma_{3}, Q\right]\right)-i e A\left[\sigma_{3}, Q \nabla Q\right]-e^{2} A^{2}\left[\sigma_{3}, Q\left[\sigma_{3}, Q\right]\right] .
$$

We now express this in cylindrical coordinates $(r, \varphi, z)$. For the term with two derivatives we get

$$
\begin{aligned}
\nabla(Q \nabla Q) & =\frac{1}{r} \partial_{r}\left(r Q \partial_{r} Q\right)+\frac{1}{r^{2}} \partial_{\varphi}\left(Q \partial_{\varphi} Q\right)+\partial_{z}\left(Q \partial_{z} Q\right) \\
& =\frac{1}{r} \partial_{r}\left(r f \partial_{r} f+r g \partial_{r} g\right)+\frac{i}{r} \partial_{r}\left(r f \partial_{r} g-r g \partial_{r} f\right) \sigma_{1} \mathrm{e}^{-i \varphi \sigma_{3}} \\
& +\frac{1}{r^{2}} \partial_{\varphi}\left(\left(g \sigma_{3}+f \sigma_{2} \mathrm{e}^{-i \varphi \sigma_{3}}\right) f \mathrm{e}^{i \varphi \sigma_{3}} \sigma_{1}\right) \\
& =\left(\frac{1}{r} \partial_{r}\left(r f \partial_{r} g-r g \partial_{r} f\right)+\frac{f g}{r^{2}}\right) i \sigma_{1} \mathrm{e}^{-i \varphi \sigma_{3}}
\end{aligned}
$$

The term $f \partial_{r} f+g \partial_{r} g$ vanishes by $f^{2}+g^{2}=1$. Taking the magnetic vector potential to be purely azimuthal, $A=e_{\varphi} A_{\varphi}(r)$, we obtain for the part linear in $A$

$$
\begin{aligned}
& -i e \nabla\left(Q\left[A \sigma_{3}, Q\right]\right)-i e\left[A \sigma_{3}, Q \nabla Q\right]=-\frac{i e}{r} \partial_{\varphi}\left(Q\left[A_{\varphi} \sigma_{3}, Q\right]\right)-\frac{i e}{r}\left[A_{\varphi} \sigma_{3}, Q \partial_{\varphi} Q\right] \\
& =-\frac{2 e A_{\varphi}}{r} \partial_{\varphi}\left(\left(g \sigma_{3}+f \sigma_{2} \mathrm{e}^{-i \varphi \sigma_{3}}\right) f \mathrm{e}^{i \varphi \sigma_{3}} \sigma_{1}\right)-\frac{i e A_{\varphi}}{r}\left[\sigma_{3},\left(g \sigma_{3}+f \sigma_{2} \mathrm{e}^{-i \varphi \sigma_{3}}\right) f \mathrm{e}^{i \varphi \sigma_{3}} \sigma_{1}\right] \\
& =-4 e A_{\varphi} \frac{f g}{r} i \sigma_{1} \mathrm{e}^{-i \varphi \sigma_{3}} .
\end{aligned}
$$

The term quadratic in the vector potential gives

$$
-e^{2} A^{2}\left[\sigma_{3}, Q\left[\sigma_{3}, Q\right]\right]=-e^{2} A_{\varphi}^{2}\left(\sigma_{3} Q \sigma_{3} Q-Q \sigma_{3} Q \sigma_{3}\right)=4 e^{2} A_{\varphi}^{2} f g i \sigma_{1} \mathrm{e}^{-i \varphi \sigma_{3}} .
$$

Gathering all terms and dividing by $i \sigma_{1} \mathrm{e}^{-i \varphi \sigma_{3}}$ we arrive at the equation

$$
\frac{1}{r} \partial_{r}\left(r f \partial_{r} g-r g \partial_{r} f\right)+\left(\frac{1}{r}-2 e A_{\varphi}\right)^{2} f g=\frac{2}{D}(|\Delta| g-i E f),
$$

whose solution for $E=0$ is discussed in the text. 


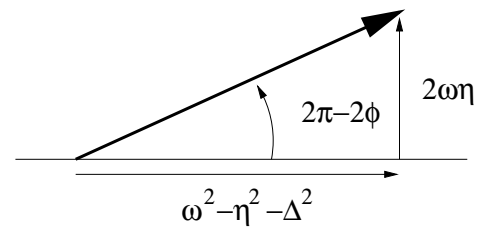

a)

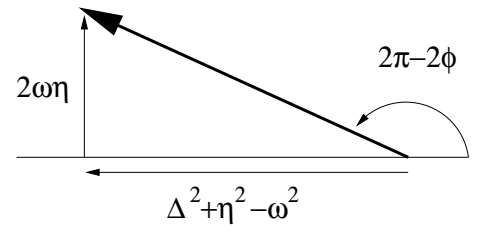

b)

Figure 4: Definition of the angle $\phi$ for a) $\omega^{2}-\eta^{2}-\Delta^{2}>0$ and b) $\omega^{2}-\eta^{2}-\Delta^{2}<0$.

\section{Choosing between solutions}

The full saddle point equation (including $\Delta$ and $\omega$ ) is difficult to solve exactly if the gap function varies in space. However, for the case of a uniform gap a solution is easy to come by. This solution is constant and describes the region far from the vortex. For values of the quasiparticle energy $\omega$ large compared to $\Delta_{0}$, the solution should approach the normal metal solution. This fact will now be used to analytically continue the metallic solution $\left(\omega / \Delta_{0} \gg 1\right)$ to the zero-energy solution for a superconductor $(\omega=0)$.

If we ignore the charge conjugation degree of freedom, which is not essential here, the full saddle point equation reads:

$$
Q(x)=\frac{i}{\pi \nu}\left\langle x\left|\frac{1}{h_{0}+i Q / 2 \tau+\sigma_{3} \tilde{\Delta}-(\omega-i \eta) \sigma_{3}}\right| x\right\rangle .
$$

In the absence of any spatial dependence in the gap $\Delta$, this is solved by Fourier transforming to momentum space and taking $Q$ to be constant:

$$
Q=\frac{i}{\pi \nu} \int \frac{d^{3} k}{(2 \pi)^{3}} \frac{1}{k^{2} / 2 m-\mu+i Q / 2 \tau+\sigma_{3} \tilde{\Delta}-(\omega-i \eta) \sigma_{3}} .
$$

Here, $\omega>0$ and $\eta>0$. We choose the gauge where $\tilde{\Delta}=\Delta \sigma_{1}$ and diagonalize the matrix $M=i \Delta \sigma_{2}-(\omega-i \eta) \sigma_{3}$ by a similarity transformation with a matrix $T$ :

$$
T M T^{-1}=\left(\begin{array}{cc}
-\sqrt{(\omega-i \eta)^{2}-\Delta^{2}} & 0 \\
0 & \sqrt{(\omega-i \eta)^{2}-\Delta^{2}}
\end{array}\right)=\lambda \sigma_{3} .
$$

The sign of the first eigenvalue, $\lambda$, of $M$ can be chosen so that $\lambda \rightarrow-\omega+i \eta$ when $\omega / \Delta \rightarrow \infty$. We then have:

$$
\lambda=\left(\left(\omega^{2}-\eta^{2}-\Delta^{2}\right)^{2}+4 \eta^{2} \omega^{2}\right)^{1 / 4} e^{i \phi},
$$

where the angle $\phi$ varies from $\pi$ to $\pi / 2$ as $\omega$ goes from infinity to 0 (see Figure $\llbracket$ ). The imaginary part of $\lambda$ remains positive for any (positive) value of $\omega$.

The matrix that diagonalizes $M$ is $T=\left(1+b \sigma_{1}\right) / \sqrt{1-b^{2}}$, with $b=-\frac{\omega-i \eta}{\Delta}+\sqrt{\left(\frac{\omega-i \eta}{\Delta}\right)^{2}-1}$. It is important that the imaginary part of $b$ is negative for any value of $\omega / \Delta$. Note in particular that for $\omega=0$ we have $b=-i$. The saddle point equation now takes the form:

$$
\bar{Q}=\frac{i}{\pi \nu} \int \frac{d^{3} k}{(2 \pi)^{3}} \frac{1}{k^{2} / 2 m-\mu+i \bar{Q} / 2 \tau+\lambda \sigma_{3}} .
$$


The physical solution is given by $\bar{Q}=T Q T^{-1}=\operatorname{sign}(\operatorname{Im} \lambda) \times \sigma_{3}=\sigma_{3}$. Therefore

$$
Q=T^{-1} \sigma_{3} T=\frac{1+b^{2}}{1-b^{2}} \sigma_{3}+\frac{2 i b}{1-b^{2}} \sigma_{2} .
$$

Unphysical solutions (which break causality) also exist.

By comparing to the ansatz used in Section 3.3 to solve the Usadel equation, $Q=\sigma_{3} \cos \theta+$ $\sigma_{2} \sin \theta$, we conclude that $b=-i \tan \frac{\theta}{2}$. In particular, for $\omega=0$ where $b=-i$, we get $\tan \frac{\theta}{2}=1$. Therefore, $\theta=\frac{\pi}{2}(\bmod 2 \pi)$ is the zero-energy solution for the homogeneous superconductor, in agreement with the asymptotics found in Section 3.3 from a different argument.

\section{E Vanishing vertices}

In this appendix we show that the terms on the second line in equation 14 do not contribute to the transmittance $\tau(E)$ in one-loop order. Let us consider the first and second term, which give

$$
\left\langle\left.\partial Z_{0}^{B 1, B 2}(0) \partial \tilde{Z}_{0}^{B 2, B 1}(1) \operatorname{STr} Z_{0} \partial \tilde{Z}_{0}\left(Z_{1} \partial \tilde{Z}_{1}-\partial Z_{1} \tilde{Z}_{1}\right)\right|_{s}\right\rangle_{0} .
$$

As before, only the contractions of type 1 and 2 in Fig. 33 are nonzero, and we obtain

$$
\mathrm{D}_{0}(\dot{0}, \dot{s})\left(\mathrm{D}_{\wedge}(\dot{s}, s)-\mathrm{D}_{\wedge}(s, \dot{s})\right) \mathrm{D}_{0}(s, \dot{1})+(\wedge \rightarrow \vee),
$$

where the dot over an argument represents a derivative acting on it. Because the first derivatives of the propagators $\mathrm{D}_{0}\left(s, s^{\prime}\right), \mathrm{D}_{\wedge}\left(s, s^{\prime}\right)$ and $\mathrm{D}_{\vee}\left(s, s^{\prime}\right)$ are discontinuous at $s=s^{\prime}$, these terms are ambiguous and have to be defined by some point-splitting procedure, e.g.

$$
\mathrm{D}_{\wedge}(\dot{s}, s)=\lim _{a \rightarrow 0} \frac{1}{a}\left(\mathrm{D}_{\wedge}\left(s+\frac{a}{2}, s\right)-\mathrm{D}_{\wedge}\left(s-\frac{a}{2}, s\right)\right)=\frac{\sinh \sqrt{i \epsilon}(1-2 s)}{2 \sinh \sqrt{i \epsilon}} .
$$

Since the propagators are symmetric in their arguments, we have

$$
\mathrm{D}_{\wedge, \vee}(\dot{s}, s)=\mathrm{D}_{\wedge, \vee}(s, \dot{s}),
$$

which establishes our claim. In the same way it is seen that the one-loop contributions from the third and fourth term in equation 14 cancel each other.

\section{F Invariant integration measure}

The field space of the nonlinear $\sigma$ model we have derived is a coset space $G / H$ with $G=$ $\operatorname{OSp}(2 n \mid 2 n)$ and $H=\mathrm{GL}(n \mid n)$. The space $G / H$ is realized by matrices

$$
q=g \Sigma_{3} g^{-1},
$$

where $g \in G$, and $\Sigma_{3}$ is fixed by the elements $h$ of the subgroup $H$ :

$$
h \Sigma_{3} h^{-1}=\Sigma_{3} .
$$

It is easily seen that the symmetry relation $q=-\gamma q^{T} \gamma^{-1}$ corresponds to the condition

$$
g=\gamma\left(g^{-1}\right)^{T} \gamma^{-1},
$$


which defines the group $G$. Let $D \mu(q)$ denote the superintegration measure (on $G / H$ ) invariant under $q \mapsto g_{0} q g_{0}^{-1}$ for all $g_{0} \in G$ and normalized by $\int D \mu(q)=1$. Our goal in this appendix is to express $D \mu(q)$ in the parametrization of $q$ introduced in Section 5.1.

Quite generally, if we are given a parametrization with (super)coordinates $\xi^{i}$ and metric tensor

$$
\mathrm{S} \operatorname{Tr}(\mathrm{d} q)^{2}=\sum_{i j} \mathrm{~d} \xi^{i} g_{i j}(\xi) \mathrm{d} \xi^{j},
$$

then the invariant integration measure is locally (i.e. modulo boundary ambiguities) expressed by

$$
D \mu(q)=D \xi \operatorname{SDet}^{1 / 2}\left(g_{i j}(\xi)\right),
$$

where $D \xi$ is a (suitably normalized) Euclidean measure. Doing the computation this way requires knowledge of all matrix elements of the metric tensor and is more complicated than is necessary.

Since we are parametrizing $q$ in the form

$$
q(\xi)=g(\xi) \Sigma_{3} g^{-1}(\xi),
$$

a more efficient approach is to calculate the measure directly from the Jacobian of the map

$$
\left(\xi^{1}, \ldots, \xi^{\operatorname{dim}}\right)=\xi \mapsto g(\xi) \Sigma_{3} g^{-1}(\xi) .
$$

However, this is still not the best method as it involves the group element $g$ twice, once on the left and once on the right (by the inverse).

The most efficient approach is to turn to the "coset space picture". Given some basis $X_{i}, \ldots, X_{\operatorname{dim}}$ of $\mathcal{P} \cong T_{H}(G / H)$, we assemble the coordinate functions $\xi^{i}$ into a matrix-valued function $X \equiv \sum_{i} \xi^{i} X_{i}: G / H \rightarrow \mathcal{P}$ and consider some map

$$
X \mapsto g(X) H,
$$

which sends $X$ into the coset space $G / H$. Its differential is

$$
\left.(X, \mathrm{~d} X) \mapsto \frac{d}{d s}\right|_{s=0} g(X+s \cdot \mathrm{d} X) H .
$$

Because the integration measure on $G / H$ we wish to express by $X$, is invariant under left translations, we may multiply by $g(X)^{-1}$ and switch to a map $T_{X}: \mathcal{P} \rightarrow \mathcal{P}$ defined by

$$
\begin{aligned}
T_{X}(\mathrm{~d} X) & =\left.\frac{d}{d s}\right|_{s=0} g(X)^{-1} g(X+s \cdot \mathrm{d} X) H \\
& =\left(\left.\frac{d}{d s}\right|_{s=0} g(X)^{-1} g(X+s \cdot \mathrm{d} X)\right)_{\mathcal{P}} .
\end{aligned}
$$

The subscript $\mathcal{P}$ means projection of a Lie algebra element $A \in \operatorname{Lie}(G)=\operatorname{Lie}(H)+\mathcal{P}$ onto $A_{\mathcal{P}} \in \mathcal{P}$. Let $J(X)=\operatorname{SDet} T_{X}$ be the Berezinian (or superjacobian) of the transformation. Then the invariant measure $D \mu(q)$ is simply expressed by

$$
D X J(X) \text {. }
$$

We now wish to apply this general formalism to the parametrization

$$
q=t_{0} t_{1} \Sigma_{3} t_{1}^{-1} t_{0}^{-1}, \quad t_{\alpha}=\left(\begin{array}{cc}
1 & Z_{\alpha} \\
\tilde{Z}_{\alpha} & 1
\end{array}\right) \quad(\alpha=0,1) .
$$


This is not possible immediately, as the matrices $t_{\alpha}$ do not obey the law $g=\gamma\left(g^{-1}\right)^{T} \gamma^{-1}$ defining the group $G=\operatorname{OSp}(2 n \mid 2 n)$ (although $q=t_{0} t_{1} \Sigma_{3} t_{1}^{-1} t_{0}^{-1}$ does satisfy $q=-\gamma q^{T} \gamma^{-1}$ ). To be on safe mathematical ground, it is advisable to start from a parametrization

$$
q=\tau_{0} \tau_{1} \Sigma_{3} \tau_{1}^{-1} \tau_{0}^{-1}
$$

such that the $\tau_{\alpha}$ do satisfy the group law. This is achieved by choosing

$$
\begin{aligned}
\tau_{\alpha} & =\left(\begin{array}{cc}
\left(1-Z_{\alpha} \tilde{Z}_{\alpha}\right)^{-1 / 2} & Z_{\alpha}\left(1-\tilde{Z}_{\alpha} Z_{\alpha}\right)^{-1 / 2} \\
\tilde{Z}_{\alpha}\left(1-Z_{\alpha} \tilde{Z}_{\alpha}\right)^{-1 / 2} & \left(1-\tilde{Z}_{\alpha} Z_{\alpha}\right)^{-1 / 2}
\end{array}\right) \\
& =\left(\begin{array}{cc}
1 & Z_{\alpha} \\
\tilde{Z}_{\alpha} & 1
\end{array}\right)\left(\begin{array}{cc}
h_{\alpha}^{+} & 0 \\
0 & h_{\alpha}^{-}
\end{array}\right)=t_{\alpha} h_{\alpha},
\end{aligned}
$$

where $h_{\alpha}^{+}=\left(1-Z_{\alpha} \tilde{Z}_{\alpha}\right)^{-1 / 2}$ and $h_{\alpha}^{-}=\left(1-\tilde{Z}_{\alpha} Z_{\alpha}\right)^{-1 / 2}$.

The general formalism now tells us

$$
D \mu(q)=D\left(Z_{0}, \tilde{Z}_{0}\right) D\left(Z_{1}, \tilde{Z}_{1}\right) J\left(Z_{0}, \tilde{Z}_{0}, Z_{1}, \tilde{Z}_{1}\right),
$$

where $D\left(Z_{0}, \tilde{Z}_{0}\right)$ and $D\left(Z_{1}, \tilde{Z}_{1}\right)$ are flat Berezin measures, $J=$ SDet $T$ is the superjacobian, and

$$
T\left(\mathrm{~d} Z_{0}, \ldots\right)=\left(\tau_{1}^{-1} \tau_{0}^{-1} \mathrm{~d}\left(\tau_{0} \tau_{1}\right)\right)_{\mathcal{P}}=\left(\tau_{1}^{-1} \mathrm{~d} \tau_{1}\right)_{\mathcal{P}}+\left(\tau_{1}^{-1}\left(\tau_{0}^{-1} \mathrm{~d} \tau_{0}\right) \tau_{1}\right)_{\mathcal{P}} .
$$

Recall the decomposition $Z=Z_{0}+Z_{1}$ which, in terms of spaces, we write $\mathcal{P}=\mathcal{P}_{0}+\mathcal{P}_{1}$. Because of $\left(\tau_{1}^{-1} \mathrm{~d} \tau_{1}\right)_{\mathcal{P}}=\left(\tau_{1}^{-1} \mathrm{~d} \tau_{1}\right)_{\mathcal{P}_{1}}$ and the general relation

$$
\operatorname{SDet}\left(\begin{array}{cc}
X & Y \\
0 & Z
\end{array}\right)=\operatorname{SDet}\left(\begin{array}{cc}
X & 0 \\
0 & Z
\end{array}\right) \text {, }
$$

we have $\operatorname{SDet} T=\operatorname{SDet} T^{\prime}$ with

$$
T^{\prime}\left(\mathrm{d} Z_{0}, \ldots\right)=\left(\tau_{1}^{-1} \mathrm{~d} \tau_{1}\right)_{\mathcal{P}_{1}}+\left(\tau_{1}^{-1}\left(\tau_{0}^{-1} \mathrm{~d} \tau_{0}\right) \tau_{1}\right)_{\mathcal{P}_{0}} .
$$

If $X$ is any element of $\operatorname{Lie}(G)$ we introduce the notation

$$
X=\left(\begin{array}{ll}
A(X) & B(X) \\
C(X) & D(X)
\end{array}\right)
$$

where $\left(\begin{array}{cc}A & 0 \\ 0 & D\end{array}\right) \in \operatorname{Lie}(H)$, and $\left(\begin{array}{cc}0 & B \\ C & 0\end{array}\right) \in \mathcal{P}$. A straightforward calculation then gives

$$
\begin{aligned}
B\left(\tau_{1}^{-1} \mathrm{~d} \tau_{1}\right) & =h_{1}^{+} \mathrm{d} Z_{1} h_{1}^{-} \\
C\left(\tau_{1}^{-1} \mathrm{~d} \tau_{1}\right) & =h_{1}^{-} \mathrm{d} \tilde{Z}_{1} h_{1}^{+}, \quad \text { and } \\
B\left(\left(\tau_{1}^{-1} \tau_{0}^{-1} \mathrm{~d} \tau_{0} \tau_{1}\right)_{\mathcal{P}_{0}}\right)= & h_{1}^{+}\left(h_{0}^{+} \mathrm{d} Z_{0} h_{0}^{-}-Z_{1} h_{0}^{-} \mathrm{d} \tilde{Z}_{0} h_{0}^{+} Z_{1}\right) h_{1}^{-}, \\
C\left(\left(\tau_{1}^{-1} \tau_{0}^{-1} \mathrm{~d} \tau_{0} \tau_{1}\right)_{\mathcal{P}_{0}}\right)= & h_{1}^{-}\left(h_{0}^{-} \mathrm{d} \tilde{Z}_{0} h_{0}^{+}-\tilde{Z}_{1} h_{0}^{+} \mathrm{d} Z_{0} h_{0}^{-} \tilde{Z}_{1}\right) h_{1}^{+} .
\end{aligned}
$$

By writing out the matrices in explicit form, one easily sees that the Berezinian of the transformation $\mathrm{d} Z_{1} \mapsto h_{1}^{+} \mathrm{d} Z_{1} h_{1}^{-}, \mathrm{d} \tilde{Z}_{1} \mapsto h_{1}^{-} \mathrm{d} \tilde{Z}_{1} h_{1}^{+}$is given by $\operatorname{SDet}\left(h_{1}^{+}\right) \operatorname{SDet}\left(h_{1}^{-}\right)=\operatorname{SDet}\left(1-\tilde{Z}_{1} Z_{1}\right)$, 
while the Berezinian of the transformation $\mathrm{d} Z_{0} \mapsto h_{0}^{+} \mathrm{d} Z_{0} h_{0}^{-}, \mathrm{d} \tilde{Z}_{0} \mapsto h_{0}^{-} \mathrm{d} \tilde{Z}_{0} h_{0}^{+}$is simply unity as a result of cancellations due to supersymmetry.

For similar reasons, the Berezinian of the transformation

$$
\begin{aligned}
\mathrm{d} Z_{0}^{\prime} & \mapsto h_{1}^{+}\left(\mathrm{d} Z_{0}^{\prime}-Z_{1} \mathrm{~d} \tilde{Z}_{0}^{\prime} Z_{1}\right) h_{1}^{-}, \\
\mathrm{d} \tilde{Z}_{0}^{\prime} & \mapsto h_{1}^{-}\left(\mathrm{d} \tilde{Z}_{0}^{\prime}-\tilde{Z}_{1} \mathrm{~d} Z_{0}^{\prime} \tilde{Z}_{1}\right) h_{1}^{+}
\end{aligned}
$$

turns out to be unity. Thus, by collecting factors the invariant integration measure $D \mu(q)$ in the parametrization $q=\tau_{0} \tau_{1} \Sigma_{3} \tau_{1}^{-1} \tau_{0}^{-1}$ is given by

$$
D\left(Z_{0}, \tilde{Z}_{0}\right) D\left(Z_{1}, \tilde{Z}_{1}\right) \operatorname{SDet}\left(1-\tilde{Z}_{1} Z_{1}\right) .
$$

Finally, we switch to the desired parametrization

$$
Q=t_{0} t_{1} \Sigma_{3} t_{1}^{-1} t_{0}^{-1}=t_{0} \tau_{1} \Sigma_{3} \tau_{1}^{-1} t_{0}^{-1}=\tau_{0} h_{0} \tau_{1} \Sigma_{3} \tau_{1}^{-1} h_{0}^{-1} \tau_{0}^{-1} .
$$

It is seen that this amounts to transforming $\tau_{1} \mapsto h_{0} \tau_{1} h_{0}^{-1}$ or, equivalently,

$$
Z_{1} \mapsto h_{0}^{+} Z_{1} h_{0}^{-}, \quad \tilde{Z}_{1} \mapsto h_{0}^{-} \tilde{Z}_{1} h_{0}^{+} .
$$

Once again, the superjacobian of this transformation is unity, so that $D \mu(q)$ in the desired parametrization has the form (15) as it stands.

In one-loop order of the perturbation expansion for the one-dimensional field theory, the factor $\operatorname{SDet}\left(1-\tilde{Z}_{1} Z_{1}\right)$ contributes a two-vertex $a^{-1} \int d s \operatorname{STr} \tilde{Z}_{1} Z_{1}$ where $a$ is the regularization

scale. This vertex does not couple to the external legs $\partial Z_{0}^{B 1, B 2}$ and $\partial \tilde{Z}_{0}^{B 2, B 1}$. Therefore we may pretend the functional integration measure to be flat, which is what we did.

\section{References}

[1] F.J. Dyson, J. Math. Phys. 3, 140 (1962).

[2] J.J.M. Verbaarschot, hep-th/9709032; hep-th/9710114.

[3] A. Altland and M.R. Zirnbauer, Phys. Rev. B55, 1142 (1997).

[4] M.A. Skvortsov and M.V. Feigel'man, Phys. Rev. Lett. 78, 2640 (1997).

[5] F.J. Wegner, Z. Phys. B35, 207 (1979).

[6] K.B. Efetov, Adv. Phys. 32, 53 (1983).

[7] K.D. Usadel, Phys. Rev. Lett. 25, 507 (1970).

[8] G. Eilenberger, Z. Phys. 182, 427 (1965); ibid. 214, 195 (1968).

[9] R.J. Watts-Tobin and G.M. Waterworth, Z. Phys. 261, 249 (1973).

[10] L. Kramer, W. Pesch, and R.J. Watts-Tobin, J. Low Temp. Phys. 14, 29 (1974).

[11] A. Altland, B.D. Simons, and J.P.D. Taras-Semchuk, JETP Lett. 67, 22 (1998).

[12] B.A. Muzykantskii and D.E. Khmel'nitskii, Phys. Rev. B51, 5840 (1995). 
[13] See, for example, P.G. De Gennes, Superconductivity of metals and alloys, Addison-Wesley, Redwood City, 1989.

[14] A. Altland, S. Iida, and K.B. Efetov, J. Phys. A26, 3545 (1993).

[15] M.R. Zirnbauer, J. Math. Phys. 37, 4986 (1996).

[16] See, for example, G. Blatter, M.V. Feigel'man, V.B. Geshkenbein, A.I. Larkin, and V.M. Vinokur, Rev. Mod. Phys. 66, 1125 (1994).

[17] K.D. Usadel, Phys. Rev. B4, 99 (1971).

[18] M.R. Zirnbauer, J. Phys. A29, 7113 (1996).

[19] M.R. Zirnbauer, Phys. Rev. Lett. 69, 1584 (1992).

[20] A.D. Mirlin, A. Müller-Groeling, and M.R. Zirnbauer, Ann. Phys. 236, 325 (1994).

[21] P.W. Brouwer and K. Frahm, Phys. Rev. B53, 1490 (1996).

[22] P.W. Brouwer and C.W.J. Beenakker, Phys. Rev. B 52, 3868 (1995).

[23] A. Altland and M.R. Zirnbauer, Phys. Rev. Lett. 76, 3420 (1996).

[24] For recent reviews see C.W.J. Beenakker, Rev. Mod. Phys. 69, 731 (1997); T. Guhr, A. Müller-Groeling, and H.A. Weidenmüller, Phys. Rep. 299, 189 (1998).

[25] K.B. Efetov and A.I. Larkin, Zh. Eksp. Teor. Fiz. 85, 764 (1983).

[26] A. Altland, B.D. Simons, and J.P.D. Taras-Semchuk, cond-mat/9807371.

[27] M.A. Skvortsov, V.E. Kravtsov, and M.V. Feigel'man, "Level statistics inside the core of a superconductive vortex", cond-mat/9805296. 